Research Article

\title{
Comparative Study of Accurate Descriptions of Hot Flow Behaviors of BT22 Alloy by Intelligence Algorithm and Physical Modeling
}

\author{
Shaoling Ding $\mathbb{D}^{1},{ }^{1}$ Chao Fang, ${ }^{1}$ and Shulin Zhang $^{2}$ \\ ${ }^{1}$ College of Science, Guilin University of Technology, Guilin 541004, China \\ ${ }^{2}$ Department of Mechanical Engineering, Liuzhou Institute of Technology, Liuzhou 545616, China \\ Correspondence should be addressed to Shaoling Ding; dingshl@glut.edu.cn
}

Received 21 October 2020; Revised 29 December 2020; Accepted 19 February 2021; Published 15 March 2021

Academic Editor: Alessandro Contento

Copyright ( 2021 Shaoling Ding et al. This is an open access article distributed under the Creative Commons Attribution License, which permits unrestricted use, distribution, and reproduction in any medium, provided the original work is properly cited.

\begin{abstract}
The nonlinear flow behaviors of BT22 alloy were investigated by thermal simulation experiments at different temperature and strain rates. Taking the experimental stress-strain data as samples, the support vector regression (SVR) model and back propagation artificial neural network (BPANN) model were established by cross-validation (CV) method to describe the nonlinear flow behaviors of BT22 alloy. Genetic algorithm (GA) was used to optimize the parameters of the SVR model and establish the GA-SVR model. At the same time, the physical model optimized by GA algorithm is compared with the machine learning model. Average absolute relative error (AARE), absolute relative error (ARE), and correlation coefficient (R) were used to evaluate the predictive ability of the four models. The results show that the order of model accuracy and generalization ability is GA-SVR > BPANN > SVR > physical model. The AARE value of the GA-SVR model is $1.5752 \%$, and the R value is as high as 0.9984, which can accurately predict the flow behaviors of BT22 alloy. According to the GA-SVR model, the flow behaviors under other conditions could be predicted to expand the experimental stress-strain data and avoid a large number of artificial tests.
\end{abstract}

\section{Introduction}

BT22 alloy is a kind of near beta titanium alloy. It has many advantages, such as high strength, high plasticity, good hardenability, and weldability, which is widely used in aircraft and other large structural load-bearing parts [1-5]. It is generally accepted that the flow stress plays an important role in many fields [6]. For example, accurate constitutive model is one of the keys to study the microdeformation mechanism and hot working characteristics of materials. A large number of experiments and related literature show that the flow stress was closely related to temperature, strain rate, and strain [7]. Thus, it is significant to establish and predict the highly nonlinear flow behaviors of BT22 alloy [8].

At present, empirical/semiempirical model, analytical model, phenomenological model, and physical model were four typical constitutive models of metal deformation at high temperature [9-12], among which Arrhenius model was the most widely used. However, these models are inferior to machine learning models in prediction accuracy and modeling [7, 11, 13-17]. Quan et al. established back propagation artificial neural network (BPANN) model and improved Arrhenius model, respectively, to study flow stress behaviors of Ti-6Al-2Zr-1Mo- $1 \mathrm{~V}$ titanium alloy. The results show that the BPANN model is better than the improved Arrhenius model [11]. Chen et al. had investigated the high temperature flow behaviors of Ti-6Al-3Nb2Zr-1Mo titanium alloy by establishing the BPANN and Arrhenius model, which showed that the accuracy of regression model was lower than BPANN [13]. Quan et al. had established BPANN and improved Arrhenius model, respectively, to predict the hot deformation behaviors of nimonic $80 \mathrm{~A}$ superalloy. It was found that the generalization ability of BPANN model was higher than the modified Arrhenius constitutive equation, which could accurately track complex characteristics of flow behaviors [14]. Li et al. had predicted the hot deformation behaviors 
of modified 2.25Cr-1Mo steel by artificial neural network (ANN) and phenomenological constitutive equations, and the results show that the ANN model was more accurate and effective than the phenomenological model [15]. The application of typical phenomenological model is limited because of low precision and complex modeling process $[18,19]$.

Therefore, the ANN, support vector machine (SVM), and other machine learning algorithms have been widely used to predict the flow stress $[20,21]$. Lin et al. had established the support vector regression (SVR) model to investigate the high temperature flow characteristics of nickel base superalloy. The SVR model could accurately describe the flow behaviors of the alloy, and in other words, the predicted results were basically consistent with the experimental results [16]. The SVR model optimized by the genetic algorithm (GA-SVR) model, ANN model, and improved Arrhenius constitutive model had been established to study the flow behaviors of Ti-6Al-2Zr-1Mo-1V alloy. The results shown that the prediction accuracy of the three models was as follows: GA-SVR $>$ ANN $>$ improved Arrhenius model, and GA-SVR had the best effect and could accurately predict the data under different test conditions [17]. The mathematical regression model, ANN, GA-SVR, and Latin hypercube sampling with SVR (LHS-SVR) were compared to establish a constitutive model of Ti-10V-2Fe$3 \mathrm{Al}$ alloy. The results shown that the predictive ability of the four models was GA-SVR $>$ LHS-SVR $>$ ANN $>$ mathematical regression model, and GA-SVR model has better efficiency [7].

ANN needs to try a variety of network topology and training parameters to achieve higher accuracy, which will consume a lot of time. For a certain dataset, trained ANN with the same network topology and parameters may obtain the fluctuation accuracy in different attempts. The BPANN model was established based on back propagation (BP) algorithm to improve accuracy. As a machine learning method based on structural risk, SVR is mainly used in the field of regression analysis, which has the advantages of strong generalization ability, strong robustness, and systematic theoretical system [16]. Compared with ANN, SVR can get global optimal solution and avoid falling into local extremum [17]. The SVR model has three parameters: penalty factor $C$, kernel parameter $g$ and insensitive loss function $\varepsilon$, which determine its learning ability and generalization ability. By optimizing the parameters, the model could predict the flow stress more accurately. The three optimal parameters of SVR had been searched by cross validation (CV) and GA to establish a stable and accurate GA-SVR model.

In this paper, the flow stress values of BT22 alloy at different deformation conditions (temperature, strain rate, and strain) were obtained through hot compression tests. SVR, GA-SVR, BPANN, and physical models had been trained to predict the flow stress according to the experimental data. The models were further evaluated by absolute mean error, correlation coefficient, and relative error. Finally, the stress-strain data of BT22 alloy were extended by using the high-precision artificial intelligence model.

\section{Materials and Methods}

The chemical compositions (wt. \%) of the adopted BT22 alloy were Al: 5.20; Mo: 4.92; V: 4.96; Cr: 1.05; Fe: 0.96, and balance Ti. The initial microstructure of BT22 alloy contained clavate primary $\alpha$, fine acicular $\alpha$ and $\beta$ matrix, as illustrated in Figure 1. The specimens were machined into cylinder bars with height $12 \mathrm{~mm}$ and diameter $8 \mathrm{~mm}$, which were lubricated with graphite to reduce the frictional effect in experiments. The samples were heated by resistance using Gleeble 3500 heating system. The test samples were heated to test temperature at a rate of $10 \mathrm{~K} / \mathrm{s}$ and held for $3 \mathrm{~min}$ to assure a uniform temperature. The samples were only compressed to a height reduction of $50 \%$, the true strain is about 0.7 , and the BT22 alloy basically reaches a steady-state flow behavior. The phase transition temperature of BT22 is about $1148 \mathrm{~K}$, and the flow behaviors of the single phase region were investigated in this paper. Therefore, four temperatures were selected in $1153-1273 \mathrm{~K}$ with an interval of $40 \mathrm{~K}$. The deformation temperatures in hot compression test were set as 1153 , 1193,1233 , and $1273 \mathrm{~K}$. The hot compression deformation rates were selected as $0.01,0.1,1$, and $10 \mathrm{~s}^{-1}$, which not only refered to some references but also represented the typical range of hot deformation rate. Compression tests were carried out at four temperatures and four deformation rates, and a total of 16 tests were carried out. The variation law of stress and strain was recorded automatically by a personal computer equipped with a data acquisition system during the compressing tests. The experimental stress-strain curves were corrected by temperature. The corrected data of BT22 alloy at various strain rates, deformation temperatures, and strains are listed in Table 1.

\section{Basic Principles}

3.1. Genetic Algorithm (GA). GA is a kind of global optimization random search algorithm which simulates biological natural selection and genetic mechanism [22-24]. It is widely used to identify and optimize model parameters in engineering due to their strong robustness, high efficiency, parallel processing, and other advantages. GA searches the global optimum in solution space by simulating the natural selection process of biological heredity and evolution. In GA, a population is regarded as a chromosome group, which is composed of some individuals representing feasible solutions. The individuals are coded which are analogous to genes. Each individual is evaluated by fitness function to get fitness value, and the corresponding individuals are inherited to the next generation. Starting from the randomly generated chromosome population, genetic algorithm performs a crossover and mutation process with fitness as the selection standard to produce a population, which is more suitable for the current environment. This group repeats continuously according to the previous generation, so as to produce the optimal individual, which is most suitable for the current environment, and thus produce the optimal feasible solution. 


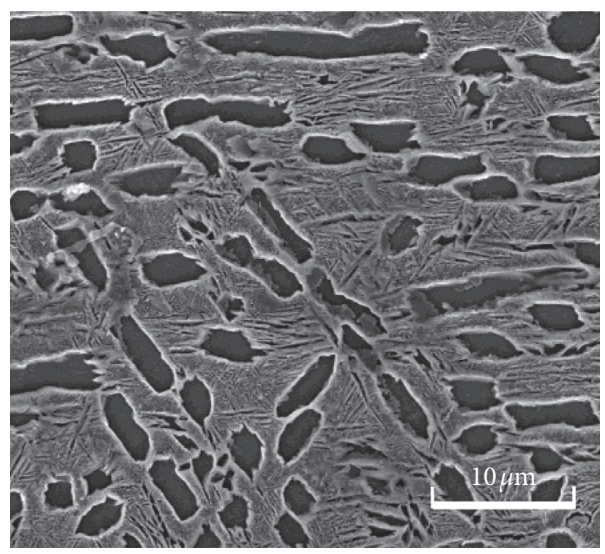

FIgURE 1: Initial microstructure of BT22 alloy.

TABLE 1: Flow stress (MPa) of BT22 alloy at different deformation conditions.

\begin{tabular}{|c|c|c|c|c|c|}
\hline \multirow{2}{*}{ Strain } & \multirow{2}{*}{ Strain rate $/ \mathrm{s}^{-1}$} & \multicolumn{4}{|c|}{ Deformation temperature/K } \\
\hline & & 1153 & 1193 & 1233 & 1273 \\
\hline \multirow{4}{*}{0.05} & 0.01 & 54.37 & 44.41 & 40.90 & 37.27 \\
\hline & 0.1 & 102.00 & 85.03 & 70.88 & 63.52 \\
\hline & 1 & 152.19 & 126.84 & 99.30 & 86.19 \\
\hline & 10 & 194.32 & 194.27 & 168.61 & 145.89 \\
\hline \multirow{4}{*}{0.10} & 0.01 & 55.93 & 45.58 & 41.61 & 37.95 \\
\hline & 0.1 & 102.70 & 86.13 & 74.31 & 69.06 \\
\hline & 1 & 157.33 & 131.85 & 108.84 & 94.38 \\
\hline & 10 & 191.99 & 197.53 & 176.06 & 151.43 \\
\hline \multirow{4}{*}{0.15} & 0.01 & 56.61 & 44.74 & 41.50 & 38.43 \\
\hline & 0.1 & 103.11 & 89.07 & 74.91 & 71.94 \\
\hline & 1 & 158.20 & 133.56 & 111.62 & 98.32 \\
\hline & 10 & 188.34 & 192.79 & 177.78 & 149.37 \\
\hline \multirow{4}{*}{0.20} & 0.01 & 57.58 & 44.18 & 40.66 & 37.59 \\
\hline & 0.1 & 103.54 & 90.57 & 75.60 & 73.23 \\
\hline & 1 & 157.77 & 136.21 & 114.82 & 99.79 \\
\hline & 10 & 189.19 & 192.31 & 176.90 & 151.06 \\
\hline \multirow{4}{*}{0.25} & 0.01 & 57.60 & 44.59 & 40.62 & 37.05 \\
\hline & 0.1 & 103.35 & 89.99 & 75.20 & 70.70 \\
\hline & 1 & 158.20 & 137.32 & 115.79 & 101.45 \\
\hline & 10 & 190.09 & 194.31 & 178.15 & 154.97 \\
\hline \multirow{4}{*}{0.30} & 0.01 & 57.93 & 44.79 & 40.20 & 36.66 \\
\hline & 0.1 & 101.17 & 88.59 & 75.26 & 66.92 \\
\hline & 1 & 157.39 & 138.39 & 116.97 & 102.02 \\
\hline & 10 & 189.61 & 191.97 & 178.54 & 152.62 \\
\hline \multirow{4}{*}{0.35} & 0.01 & 57.44 & 43.93 & 39.72 & 36.13 \\
\hline & 0.1 & 99.46 & 85.30 & 73.76 & 64.28 \\
\hline & 1 & 155.96 & 138.14 & 117.25 & 102.02 \\
\hline & 10 & 189.30 & 192.66 & 178.71 & 153.42 \\
\hline \multirow{4}{*}{0.40} & 0.01 & 56.71 & 43.36 & 39.33 & 35.66 \\
\hline & 0.1 & 97.95 & 84.07 & 72.67 & 63.76 \\
\hline & 1 & 154.42 & 137.99 & 116.94 & 101.81 \\
\hline & 10 & 188.19 & 189.46 & 178.37 & 153.14 \\
\hline \multirow{4}{*}{0.45} & 0.01 & 55.72 & 42.69 & 38.92 & 35.62 \\
\hline & 0.1 & 96.32 & 83.80 & 71.07 & 63.41 \\
\hline & 1 & 153.20 & 136.21 & 116.75 & 101.54 \\
\hline & 10 & 187.79 & 188.58 & 175.83 & 153.38 \\
\hline
\end{tabular}


TABLE 1: Continued.

\begin{tabular}{|c|c|c|c|c|c|}
\hline \multirow{2}{*}{ Strain } & \multirow{2}{*}{ Strain rate $/ \mathrm{s}^{-1}$} & \multicolumn{4}{|c|}{ Deformation temperature/K } \\
\hline & & 1153 & 1193 & 1233 & 1273 \\
\hline \multirow{4}{*}{0.50} & 0.01 & 54.76 & 42.38 & 38.40 & 35.44 \\
\hline & 0.1 & 95.00 & 83.24 & 70.24 & 62.58 \\
\hline & 1 & 151.67 & 135.65 & 115.74 & 100.47 \\
\hline & 10 & 186.31 & 186.48 & 172.72 & 152.01 \\
\hline \multirow{4}{*}{0.55} & 0.01 & 53.82 & 41.77 & 38.48 & 34.95 \\
\hline & 0.1 & 93.24 & 82.31 & 68.75 & 61.05 \\
\hline & 1 & 149.76 & 135.05 & 115.06 & 100.13 \\
\hline & 10 & 185.89 & 186.22 & 170.21 & 151.12 \\
\hline \multirow{4}{*}{0.60} & 0.01 & 53.24 & 41.38 & 36.94 & 34.49 \\
\hline & 0.1 & 91.23 & 81.40 & 67.92 & 59.78 \\
\hline & 1 & 147.90 & 132.84 & 112.84 & 98.05 \\
\hline & 10 & 184.11 & 183.98 & 168.58 & 149.90 \\
\hline
\end{tabular}

3.2. Cross Validation (CV). $\mathrm{CV}$ is a practical method to cut data samples into smaller subsets in order to obtain reliable and stable models, also known as cyclic estimation [25]. Three cross validation methods are well known: $k$-fold, holdout, and leave-one-out. The $k$-fold method was adopted in this paper. Firstly, the training dataset $S$ was randomly divided into $k$ mutually exclusive subsets with the same size, that is, $S=S 1 \cup S 2 \cdots \cup S k, S i \cap S j=\Phi(i \neq j)$. Then, $k-1$ subsets were randomly selected as training dataset in each time, and the remaining one was used as test dataset. After cross validation was repeated $k$ times, the optimal model and parameters of loss function were selected.

3.3. Support Vector Regression (SVR). SVR is an artificial intelligence algorithm based on statistical learning, which is an important application branch of SVM [26]. SVR had been widely and successfully used in regression analysis of many nonlinear engineering problems [27-29]. The main idea is to map samples to high-dimensional feature space by kernel function and find the maximum boundary hyperplane to fit them.

SVR model maps the specified observation variables $T=$ $\left\{\left(x_{1}, y_{1}\right), \ldots,\left(x_{i}, y_{i}\right), \ldots,\left(x_{n}, y_{n}\right)\right\}$ to high-dimensional feature space (Hibert space). The input variables mapped to high-dimensional feature space are used for linear regression to form support vector regression hyperplane, which are transformed from the nonlinear function model to the linear function model, as follows:

$$
F\left(X_{i}\right)=b+W^{T} X_{i}
$$

where $F\left(X_{i}\right)$ is a linear function of SVR hyperplane; $b$ is a constant term; $W^{T}$ is the weight; and $X_{i}$ is the nonlinear mapping.

The SVR model, like the classical regression model, should follow the principle of minimum loss function. In order to avoid overfitting, a quadratic $\mathcal{\varepsilon}$-insensitive loss function was introduced under the assumption of hyperplane parameter estimation. When the absolute error between the experimental data and the fitted data by model was less than the preintroduced $\varepsilon$, it was considered that the loss function contribution of the observed value is 0 , which was called the "insensitive response" of the loss function. If the absolute error was greater than $\varepsilon$, the contribution and loss function of the observation loss function as follows:

$$
\begin{aligned}
L_{i}^{\varepsilon} & =\left(\max \left(0,\left(e_{i}-\varepsilon\right)^{2}\right),\right. \\
L^{\varepsilon} & =\sum_{i=1}^{n} L_{i}^{\varepsilon} .
\end{aligned}
$$

The weight $W$ and constant term $b$ of regression function were found by the $\varepsilon$-insensitive loss function algorith and structural risk minimization (SRM) principle [30]. Then, the optimization function could be obtained as follows:

$$
\begin{aligned}
& \min , L=\frac{1}{2}\|W\|^{2}+C \sum_{i=1}^{n}\left(\zeta_{i}+\zeta_{i}^{*}\right), \\
& \text { s.t., }\left\{\begin{array}{l}
y_{i}-\left(W X_{i}+b\right) \leq \varepsilon+\zeta_{i}^{*}, \quad i=1,2, \ldots, n, \\
\left(W X_{i}+b\right)-y_{i} \leq \varepsilon+\zeta_{i}, \quad i=1,2, \ldots, n, \\
\zeta_{i}, \zeta_{i}^{*} \geq 0, \quad i=1,2, \ldots, n,
\end{array}\right.
\end{aligned}
$$


where $\zeta_{i}$ and $\zeta_{i}^{*}$ are relaxation variables and $C$ is penalty parameter. Under the condition of Karush-Kuhn-Tucker (KKT), the partial derivatives for $b, W$, and $\zeta_{i}^{*}$ in the constructed Lagrange function are calculated. When the gradient is equal to 0 , the optimization problem is transformed into a dual problem, which is also called $2 n$ order quadratic programming:

$$
\begin{array}{ll}
\min & L=\frac{1}{2} \sum_{i, j=1}^{n}\left(\partial_{i}^{*}-\partial_{i}\right)\left(\partial_{j}^{*}-\partial_{j}\right)\left(x_{i} \cdot x_{j}\right)+\varepsilon \sum_{i=1}^{n}\left(\partial_{i}^{*}+\partial_{i}\right)-\sum_{i=1}^{n} y_{i}\left(\partial_{i}^{*}-\partial_{i}\right), \\
\text { s.t. } & \sum_{i=1}^{n}\left(\partial_{i}^{*}-\partial_{i}\right)=0, \quad 0 \leq \partial_{i}, \partial_{i}^{*} \leq C, \quad i=1, \ldots, n,
\end{array}
$$

where $\left(x_{i} \cdot x_{j}\right)$ is the vector dot product of the sample variable and $\partial_{i}^{*}-\partial_{i}$ is the parameter value corresponding to the support vector.

For the inner product problem satisfying Mercer's theorem in high-dimensional feature space, a kernel function $K\left(x_{i} \cdot x_{j}\right)$ is constructed to replace the above formula $\left(x_{i} \cdot x_{j}\right)[31,32]$. Then, the objective function is transformed into a linear function regression problem, which makes the solution more concise and convenient [20]:

$$
\begin{array}{ll}
\min & L=\frac{1}{2} \sum_{i, j=1}^{n}\left(\partial_{i}^{*}-\partial_{i}\right)\left(\partial_{j}^{*}-\partial_{j}\right) K\left(x_{i} \cdot x_{j}\right)-\sum_{i=1}^{n} y_{i}\left(\partial_{i}^{*}-\partial_{i}\right)+\varepsilon \sum_{i=1}^{n}\left(\partial_{i}^{*}+\partial_{i}\right), \\
\text { s.t. } & \sum_{i=1}^{n}\left(\partial_{i}^{*}-\partial_{i}\right)=0, \quad 0 \leq \partial_{i}, \partial_{i}^{*} \leq C, \quad i=1, \ldots, n,
\end{array}
$$

where $K\left(x_{i} \cdot x_{j}\right)$ represents a radial basis kernel function. The SMO algorithm proposed by John is used to solve the above equation, and the optimal solution of convex programming problem is obtained. Then, SVR model can be solved as follows:

$$
\begin{aligned}
F\left(X_{i}\right) & =b+\sum_{i=1}^{n}\left(\partial_{i}^{*}-\partial_{i}\right) K\left(x_{i} \cdot x_{j}\right), \\
K\left(x_{i} \cdot x_{j}\right) & =\exp \left(-g\left\|x_{i}-x_{j}\right\|^{2}\right),
\end{aligned}
$$

where $g$ represents the parameter value of kernel function. So, $b$ can be calculated by the following formula:

$$
b=y_{j}-\sum_{i=1}^{n}\left(\partial_{i}^{*}-\partial_{i}\right) K\left(x_{i} \cdot x_{j}\right) \pm \varepsilon, \quad \partial_{i}^{*}, \partial_{i} \in(0, C) .
$$

3.4. BPANN. ANN is a new intelligent algorithm of machine learning and is widely used in data classification, clustering, and regression prediction. In 1986, Rumelhart, Hinton, and Williams proposed a complete and concise ANN error back propagation (BP) algorithm, which systematically solved the learning problem of hidden unit link weight in a multilayer network [33]. BPANN is a multilayer feed forward neural network whose topology includes input layer, hidden layer, and output layer. The BPANN learning process consists of forward calculation process and error back propagation process. In the forward process, the output signal is calculated from the input layer through the hidden layer nodes and finally transmitted to the output layer. The state of the next layer of neurons is only affected by the state of the neurons in the upper layer. If the output layer cannot get the expected output signal, it will enter the process of error back propagation. The error signal returns layer by layer along the original connection signal until it reaches the input layer. The weight and threshold are adjusted layer by layer, and the calculation is repeated. Through these two processes, the weights and thresholds of each layer are continuously adjusted to minimize the network error, and the model training is completed. The structure of BPANN network with a single hidden layer is shown in Figure 2.

In the perceptron model above, the input vector is $X$, $X=\left(x_{1}, x_{2}, \ldots, x_{i}, \ldots, x_{n}\right)^{T}$; the input vector of hidden layer is $Y, Y=\left(y_{1}, y_{2}, \ldots, y_{j}, \ldots, y_{m}\right)^{T}$; the output vector of the output layer is expressed by $O, O=\left(o_{1}, o_{2}, \ldots, o_{k}, \ldots, o_{l}\right)^{T}$; and expected output vector is expressed by $d$, $d=\left(d_{1}, d_{2}, \ldots, d_{k}, \ldots, d_{l}\right)^{T}$. The weight matrix between the input layer and hidden layer is expressed by $V$, $V=\left(V_{1}, V_{2}, \ldots, V_{j}, \ldots, V_{m}\right)$, where column vector $V_{j}$ is the weight vector corresponding to the $j$ th neuron in the hidden layer; the weight matrix between the hidden layer and the output layer is expressed by $W, W=\left(w_{1}, w_{2}, \ldots, w_{k}, \ldots, w_{l}\right)$, where column vector $w_{k}$ is the weight vector corresponding to the $k$ th neuron in the output layer. The graph shows the number of input layer neuron nodes $n$, hidden layer neuron nodes $m$, and output layer nodes $l$. This structure is called the three-layer BP network of $n-m-l$. 


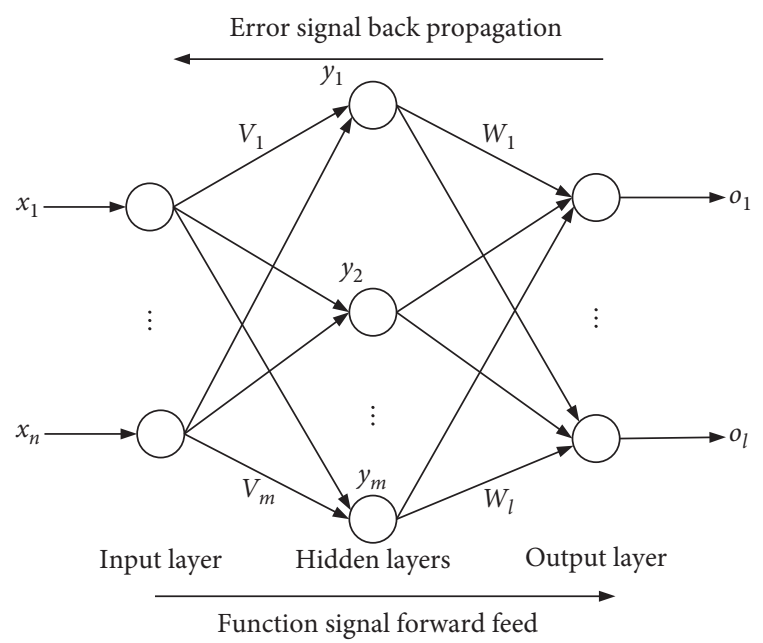

FIgURE 2: Topological structure of the BPANN network with a single hidden layer.

The following describes the implementation of the BPANN model. There are $p$-pairs of training samples, and then the input vector $X^{p}$ and the expected output vector $d^{P}$ are assigned to the vector groups $X$ and $d$. Therefore, the output vectors $Y$ and $O$ can be calculated by the following formulae:

$$
\begin{aligned}
& y_{j}=f\left(V_{j}^{T} X\right), \quad j=1,2, \ldots, m, \\
& o_{k}=f\left(W_{k}^{T} Y\right), \quad k=1,2, \ldots, l .
\end{aligned}
$$

The output error is calculated by the following formulae. Since $p$-pairs of training samples have different output error $E^{P}$ (equation (11)), it can be concluded that the root mean square error of all samples is the total output error $E$ (equation (12)).

$$
\begin{aligned}
E^{p} & =\sqrt{\sum_{k=1}^{l}\left(d_{k}^{p}-o_{k}^{p}\right)^{2}} \\
E & =\sqrt{\frac{1}{p} \sum_{p-1}^{p}\left(E^{p}\right)^{2}}
\end{aligned}
$$

The error signal $\delta_{k}^{o}(13)$ is obtained by comparing the expected output $d$ of the output layer with the actual output $O$ and then is propagated back to each node of the hidden layer to obtain the hidden layer error signal $\delta_{j}^{y}(14)$. The weights $W$ and $V$ of each layer are calculated and adjusted by getting the error signals of each layer, and the rules are formulae (15), (16), where $\beta$ is the learning rate and its value is the decimal of $(0,1]$.

$$
\begin{aligned}
& \delta_{k}^{o}=\left(d_{k}-o_{k}\right)\left(1-o_{k}\right) o_{K}, \quad k=1,2, \ldots, l, \\
& \delta_{j}^{y}=\left(\sum_{k=1}^{l} \delta_{k}^{o} W_{j k}\right)\left(1-y_{j}\right) y_{j}, \quad j=1,2, \ldots, m,
\end{aligned}
$$

$$
\begin{gathered}
w_{j k} \Leftarrow w_{j k}+\beta \delta_{k}^{o} y_{j}, \quad k=1,2, \ldots, l ; j=0,1, \ldots, m, \\
v_{i j} \Leftarrow v_{i j}+\beta \delta_{j}^{y} x_{i}, \quad j=1,2, \ldots, m ; x=0,1, \ldots, n .
\end{gathered}
$$

3.5. Physical Model. From the perspective of physical, the whole hot deformation process of alloy can be divided into two parts: elastic and plastic sections. Therefore, the flow stress can be described as follows:

$$
\sigma=\sigma_{a}+\sigma_{b}
$$

where $\sigma$ is the flow stress, $\sigma_{a}$ is the elastic stress, and $\sigma_{b} \sigma_{b}$ is the represents plastic stress which is relevant to dislocation density evolution.

In general, the stress at $0.2 \%$ plastic deformation is defined as the elastic stress. The elastic stresses are mainly affected by strain rate and temperature. The relationship between the parameters of equation and elastic stress can be expressed as follows [34, 35]:

$$
\sigma_{a}=A *\left(e * \exp \left(\frac{Q_{1}}{R * T}\right)\right)^{B},
$$

where $A, B$, and $Q_{1}$ are the estimated parameters, $e$ is the strain rate, and $T$ is the deformation temperature.

The plastic stress generally varies with the dislocation density, and the quantitative relationship between them can be described as follows [36]:

$$
\begin{aligned}
\sigma_{b} & =M * \tau, \\
\tau & =a * b * u * \sqrt{\rho},
\end{aligned}
$$

where $M$ stands for Taylor factor (3.06), $a$ stands for constant (0.5), $\rho$ stands for dislocation density, $\tau$ stands for resolved shear stress, $b$ stands for Burgers vector $\left(2.86 * 10^{-10} \mathrm{~m}^{-1}\right), u$ stands for the elastic modulus related to temperature, and the specific relationship can be expressed as follows [37]:

$$
u=-15.3 * T+21884.7 \mathrm{MPa} .
$$

According to the $\mathrm{K}-\mathrm{M}$ model of dislocation evolution, work hardening leads to dislocation multiplication, and the dislocation density increment can be measured by $1 /(b * c)$. Dynamic softening leads to dislocation annihilation, which is proportional to the dislocation, and the scaling coefficient is $K_{b}$ [38-40]. Therefore, the change rate of dislocation density with strain can be expressed by

$$
\frac{\partial \rho}{\partial w}=\frac{1}{(b * c)}-K_{b} \rho,
$$

where $b$ is the Burgers vector, $c$ is the average free path of dislocation, $K_{b}$ is the material constant, and $\rho$ is dislocation density.

Grain size (R), substructure (s), precipitates, and others are the main factors affecting the average free path of dislocations. It can be expressed as follows [41]: 


$$
\frac{1}{c}=\frac{1}{R}+\frac{1}{s}+\frac{1}{\text { others }}
$$

On the one hand, the stress-strain curves of TB22 alloy during hot deformation in the $\beta$-phase region presented the characteristics of steady-state curve, and the steady-state flow occurred when flow stress reached the peak value. It was considered that dynamic recovery was the main softening mechanism. On the other hand, when the deformation activation energy was close to the self-diffusion activation energy of pure titanium, the softening mechanism in this phase region was mainly dynamic recovery. On the contrary, the softening mechanism was mainly dynamic recrystallization [42]. The deformation activation energy of the BT22 alloy in the $\beta$ region was calculated by Arrhenius constitutive equation, which was about $183-222 \mathrm{~kJ} \cdot \mathrm{mol}^{-1}$. Generally, the selfdiffusion activation energies of $\beta$-Ti and $\alpha$-Ti were 153 and $169 \mathrm{~kJ} \cdot \mathrm{mol}^{-1}$, respectively [43]. The deformation activation energy was slightly greater than the self-diffusion activation energies of $\beta$-Ti, and there was a small difference. Based on the above analysis, the main mechanism of hot deformation in $\beta$-phase region of BT22 alloy was dynamic recovery.

The main softening mechanism of BT22 alloy is DRV, and there is no phase transformation and precipitation in the $\beta$ region during the experiment. Therefore, equation (22) can be simplified to equation (23).

$$
\frac{1}{c}=\frac{1}{s}
$$

According to references [44-46], the formation and evolution of substructure is inversely proportional to the square root of $\rho$, and the following formula can be derived:

$$
s=\frac{K_{a}}{\sqrt{\rho}}
$$

where $K_{a}$ is material constants related to strain rate $(e)$ and temperature $(T)$, which is similar to $K_{b}$. They can be described by $f$ as follows:

$$
\begin{aligned}
& K_{b}=A_{b} *\left(e * \exp \left(\frac{Q_{b}}{(R * T)}\right)\right)^{n_{b}}, \\
& K_{a}=A_{a} *\left(e * \exp \left(\frac{Q_{a}}{(R * T)}\right)\right)^{n_{a}},
\end{aligned}
$$

where $A_{b}, A_{a}, Q_{b}, Q_{a}, n_{b}$, and $n_{a}$ are the estimated parameters, $e$ is the strain rate, and $T$ is the deformation temperature.

Substituting equations (23) and (24) into (21), the following equation can be obtained.

$$
\frac{\partial \rho}{\partial w}=\frac{1}{b} * \frac{1}{K_{a}} \sqrt{\rho}-K_{b} \rho .
$$

\section{The Stress Prediction Model}

4.1. The Stress Prediction Model Based on GA-SVR. 192 inputoutput pairs in Table 1 were divided randomly into $70 \%$ and $30 \%$. 134 pairs were randomly selected to train the model, and then the rest 58 pairs were used to verify the generalization ability of the model. These data were unified to reduce error and improve the model accuracy. In this paper, the maximum and minimum standardization was used, so that data were controlled in the range of $[0,1]$. The normalization function of the dataset is expressed as

$$
x_{i}^{*}=\frac{\left(x_{i}-x_{\min }\right)}{\left(x_{\max }-x_{\min }\right)},
$$

where $x_{i}$ represents the experimental value in the original dataset, $x_{\min }$ represents the minimum, $x_{\max }$ represents the maximum, and $x_{i}^{*}$ is the normalized data of the corresponding $x_{i}$.

The prediction accuracy of SVR model mainly depends on penalty factor parameters $(C)$, kernel function parameters $(g)$, and insensitive loss parameters $(\varepsilon)$. GA was used to efficiently search the optimal parameter combination of the three parameters $(C, g$, and $\varepsilon$ ). The SVR model of BT22 alloy flow stress was optimized by combining 5-fold cross validation and GA algorithm. Therefore, a more accurate GASVR model can be established, and the detailed flowchart is shown in Figure 3.

In the first step, the original dataset was divided into verification dataset and training dataset and was standardized. In the second step, three parameters $(C, g$, and $\varepsilon$ ) to be optimized were coded on the chromosome of the individual to initialize the population of GA-SVR with the cross-validation model. During the solution process, the population number was 50 and the number of iterations was 200. In the third step, the training set was randomly divided into 5 groups and the SVR model was trained 5 times using cross validation. The mean square error (MSE) of 5 training results was used as the total MSE. In the fourth step, the fitness value of the individual was calculated by using 1/MSE index as shown in formula (18). Individual fitness values were sorted according to size. Individuals with higher fitness were selected for retention, and individuals with less fitness were excluded. In the fifth step, if the iteration termination condition was not satisfied, the chromosomes of individuals with higher fitness value were selected, crossed, and mutated to obtain the new species group, and then the third step was repeated. Otherwise, the iteration was stopped, and the optimization model parameters were obtained. In the sixth step, the optimal combination of output parameters was brought into the model to test or verify the model, and the predicted values were the output.

The fitness function is expressed as follows:

$$
\frac{1}{\operatorname{MSE}}=\frac{1}{\left((1 / n) \sum_{i=1}^{n}\left[f\left(x_{i}\right)-y_{i}\right]^{2}\right)},
$$

where $y_{i}$ is the experimental value; $f\left(x_{i}\right)$ is the predicted value; and $n$ is the number of experimental samples.

The population fitness function curve is shown in Figure 4(a). When the number of iterations was 200, the optimal fitness value was extremely close to the average fitness value. The optimal parameters of the SVR model were $C=99.66783, g=10.06612$, and $\varepsilon=0.001498$, respectively. 


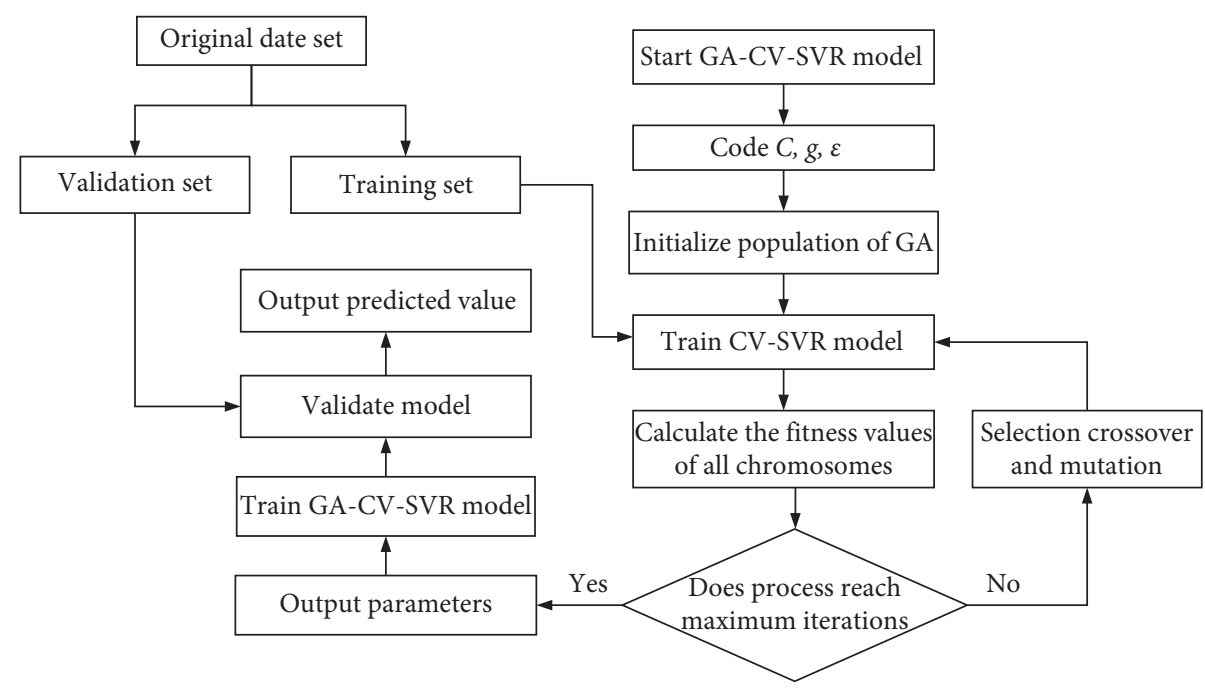

FIGURE 3: Detailed flowchart of the GA-SVR prediction model.

According to previous experience and statistical theory, three classical evaluation indexes including correlation coefficient (R), absolute relative error (ARE), and average absolute relative error (AARE) were applied to assess accuracy and relevance between the experimental values and predicted values, which were expressed as equations (29) and (31), respectively. In common, a larger $R$ value and a lesser ARE value and AARE value indicated that the solved model had a higher accuracy level.

$$
\begin{gathered}
R=\frac{\sum_{i=1}^{n}\left(y_{i}-\bar{y}_{i}\right)\left(f\left(x_{i}\right)-\overline{f\left(x_{i}\right)}\right)}{\sqrt{\sum_{i=1}^{n}\left(y_{i}-\bar{y}_{i}\right)^{2} \sum_{i=1}^{n}\left(f\left(x_{i}\right)-\overline{f\left(x_{i}\right)}\right)^{2}}} \\
\mathrm{ARE}=\left|\frac{y_{i}-f\left(x_{i}\right)}{y_{i}}\right| \\
\mathrm{AARE}=\frac{1}{n} \sum_{i=1}^{n}\left|\frac{y_{i}-f\left(x_{i}\right)}{y_{i}}\right| \times 100 \%,
\end{gathered}
$$

where $y_{i}$ is the experimental data; $f\left(x_{i}\right)$ is the predicted data; $\overline{y_{i}}$ is the average values of experimental data; and $\overline{f\left(x_{i}\right)}$ is the average values of predicted data.

Figure 4(b) shows the scatter diagram of the experimental data and the predicted data. It could be seen that the predicted data were basically consistent with experimental data, indicating that the prediction effect was good. Figure 4(c) shows the scatter diagram of the experimental data and the predicted data in the training set, and the data points were basically located on the best fitting line. The $R$ value and AARE value were 0.9998 and $0.8388 \%$, respectively, indicating that the model fitting effect was good. Figure 4(d) shows the scatter plot of the experimental data and the predicted data in the validation data set. It could be seen that the predicted values were basically in a straight line with the experimental values. The $R$ value of model validation was 0.99841 , and AARE value was $1.5752 \%$. Compared with Figures $4(\mathrm{c})$ and $4(\mathrm{~d})$, the $R$ value had little difference, and the AARE value had increased by $87.7921 \%$, but it still remained below $2 \%$, indicating that the prediction stability of the model was good. From Figures 4(e) and 4(f), $96.2687 \%$ of the ARE values of the training set were less than $4 \%$, and $94.8276 \%$ of the ARE values of the validation set were less than $4 \%$. Therefore, it could be seen that most of the ARE values were within $4 \%$, indicating that the prediction error was small and the prediction accuracy of the model was high.

4.2. The Stress Prediction Model Based on BPANN. BPANN model is a highly nonlinear system with strong generalization and fault tolerance ability, which is suitable for nonlinear data prediction. The original data were normalized and randomly divided into training dataset and verification dataset according to the ratio of $7: 3$. The BPANN model was trained by the 5 -fold cross validation method. Taking the mean square root of the total output error of all samples as the final total output error was the objective function. The detailed flowchart of establishing BPANN model is shown in Figure 5.

Firstly, the experimental dataset was divided into training dataset and verification dataset and then was standardized. The weight of the network was initialized, and the model was trained by 5 -fold cross validation. Secondly, the output error of each layer and the total output error were calculated. The fitness function was expressed as equation (12). Thirdly, the weight was dynamically adjusted based on the error signal. Fourthly, if the final error of the network satisfied the accuracy requirements or the number of iterations reached the set requirements, the training was over. Otherwise, step 2 would return. Fifthly, the model was established, and the prediction and validation data were output.

The number of hidden layers can be arbitrary in the BPANN model. When the number of hidden layers increases, the training accuracy of the model is improved, but at the same time, the training time of the model increases and the generalization ability of the network decreases. The 


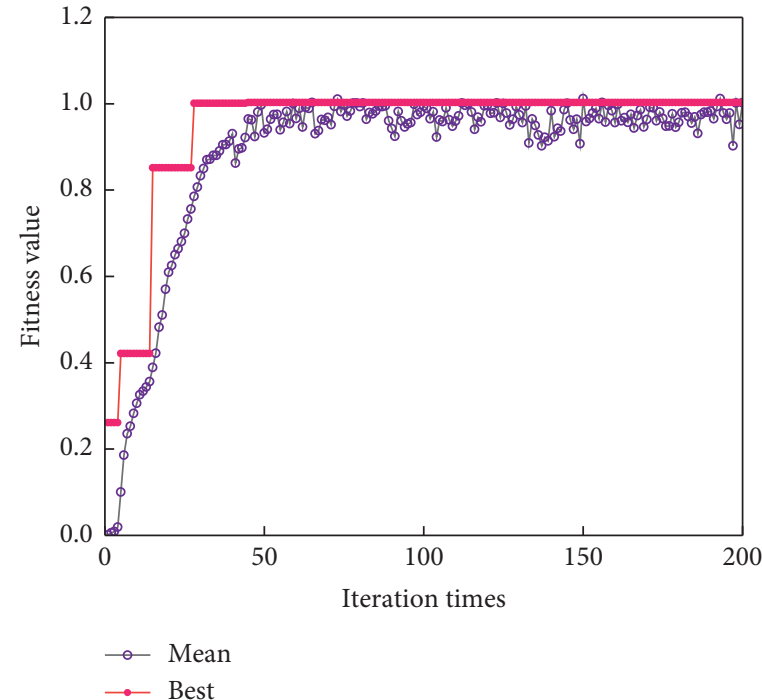

(a)

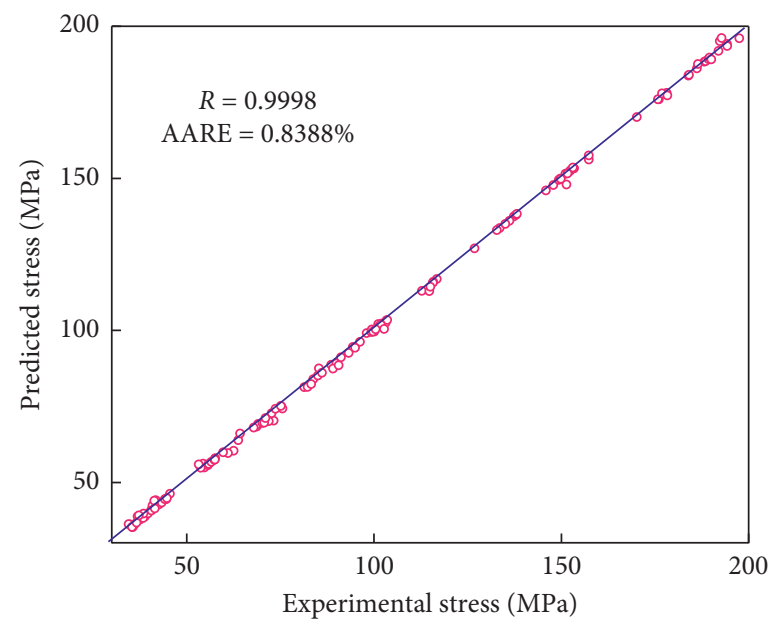

- Training data points

(c)

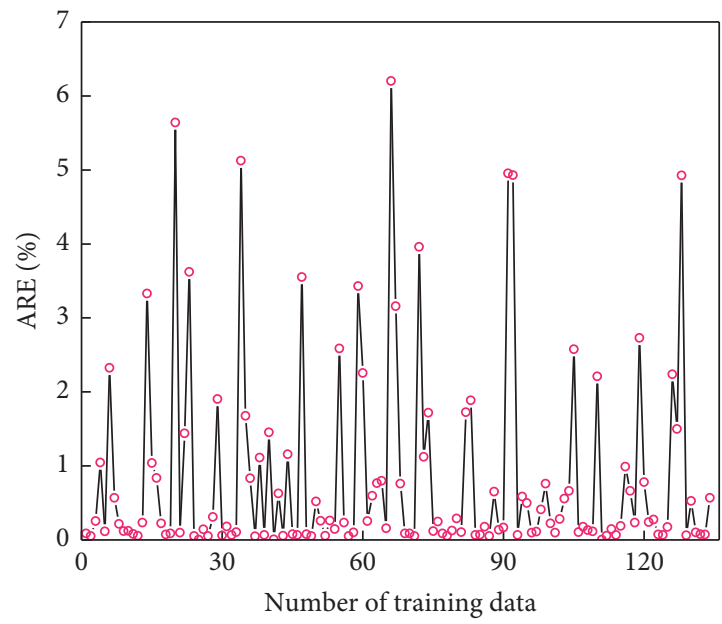

(e)

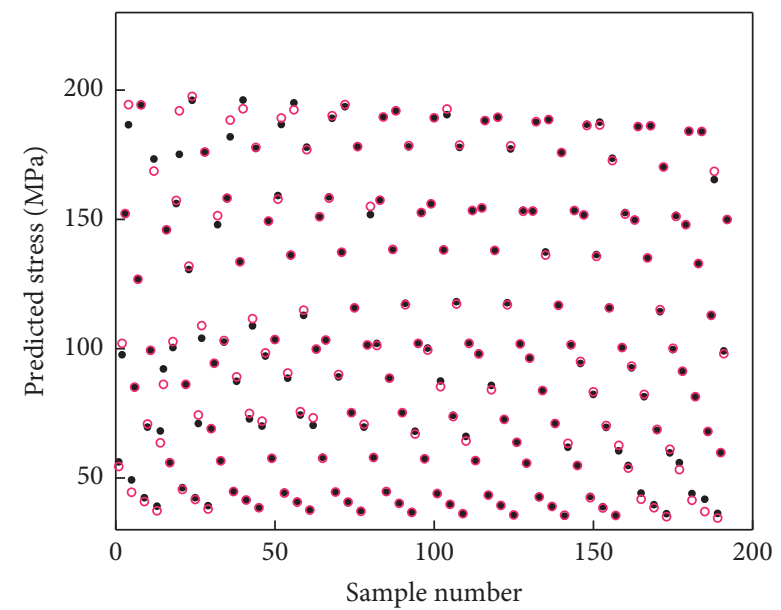

- Predicted stress

- Experimental stress

(b)

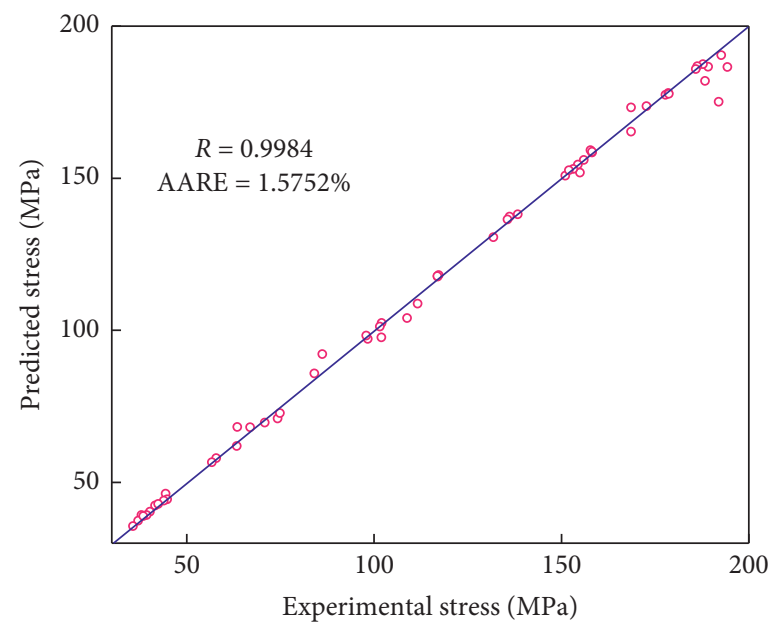

- Validation data points

(d)

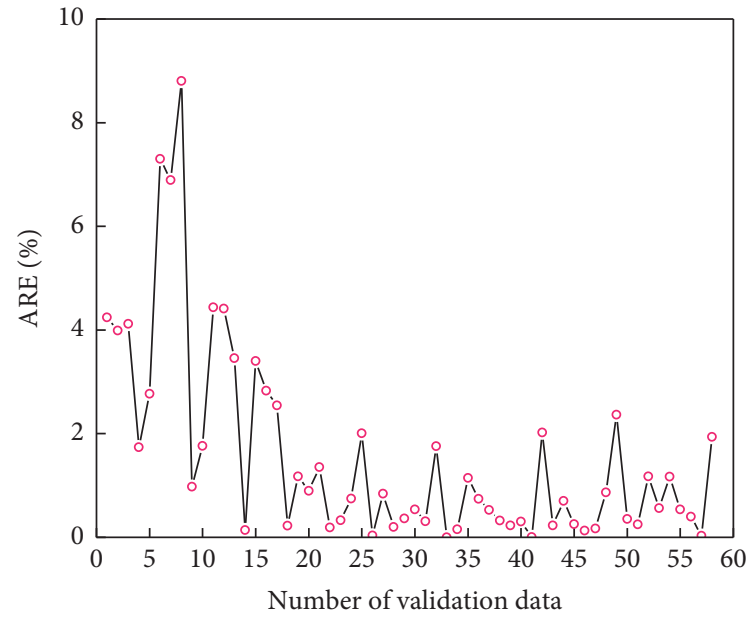

(f)

FIgURE 4: Correlations between the experimental stress and predicted stress by GA-SVR. (a) Fitness values varies with iterations. (b) All data. (c) Training data. (d) Validation data. (e) ARE in training. (f) ARE in validation. 


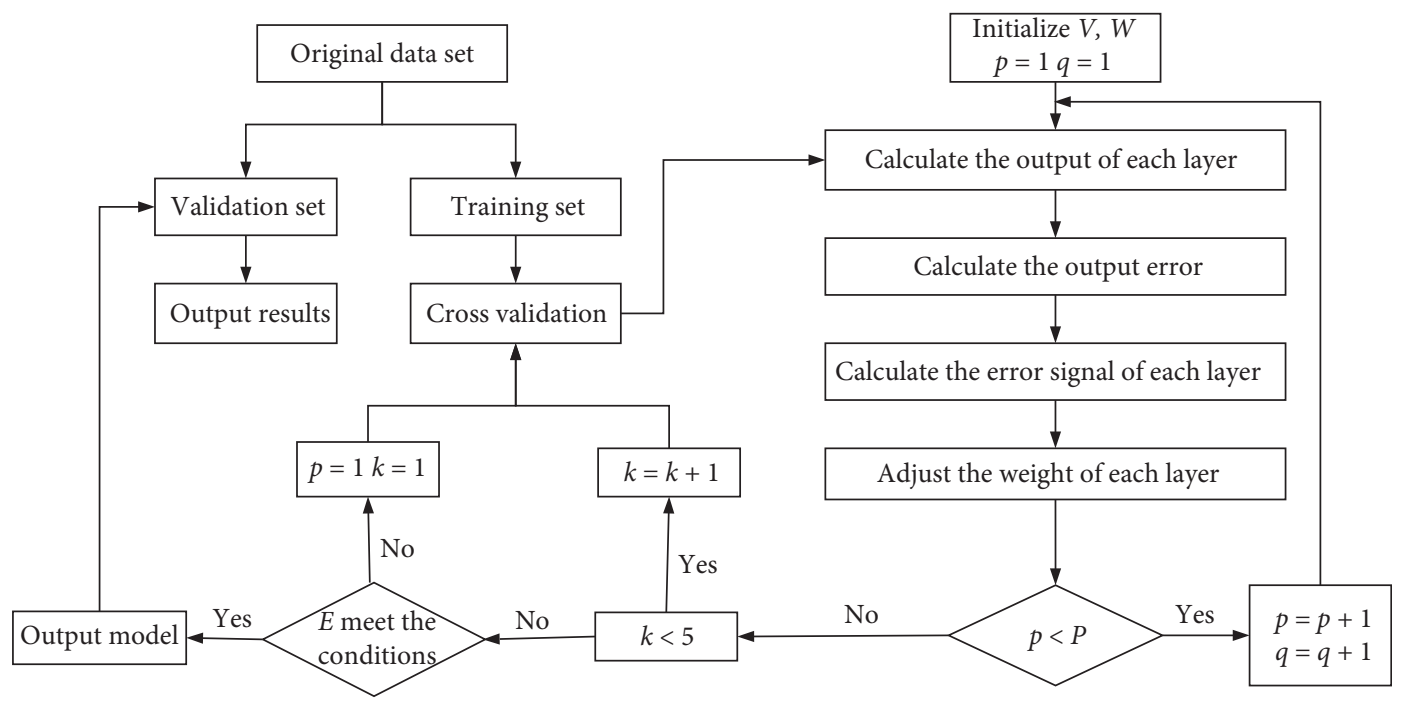

FIgURE 5: Detailed flowchart of the BPANN prediction mode.

number of hidden layers should be minimized, and the forecast accuracy should be controlled by number of hidden layer nodes. Therefore, the BPANN model often uses the single hidden layer structure. The number of input nodes is relatively small, the number of hidden layer nodes can be determined according to the input layer nodes, and the specific method is as follows:

$$
n_{1}=\frac{1}{2} n_{2}, n_{2}, 2 n_{2}, 2 n_{2}+1
$$

where $n_{2}$ is the number of input nodes and $n_{1}$ is the number of hidden nodes. The appropriate number of hidden layer nodes may be 2, 3, 6, and 7. Therefore, four kinds of BPANN models were established, including 3-2-1, 3-3-1, 3-6-1, and 3-7-1. The appropriate BPANN model was determined as $3-7-1$ by comparing the prediction accuracy of each model. The hidden layer function selects sigmoid.

Figure 6(a) shows a scatter plot of predicted and experimental values. Most of the predicted values overlapped with the experimental values, indicating that the predicted data were good. Figure 6(b) shows a scatter plot of the predicted and experimental data in the training data. It could be seen that most of the predicted data were in the same line with the experimental data, which show that the training data fitted well. The $R$ value and AARE value of the training model were 0.9996 and $1.4424 \%$, respectively. Figure 6(c) shows that the $R$ value and AARE value of validation dataset were 0.9980 and $2.4351 \%$, respectively. Although the accuracy of validation dataset was lower than training set, it still had high prediction accuracy. As could be seen from Figures 6(d) and 6(e), the ARE values of the training dataset and validation dataset of the BPANN model are not more than $4 \%$, accounting for $91.7910 \%$ and $84.4827 \%$, respectively. Therefore, it could be seen that most of the ARE values were within 4\%, which indicated that the forecast error was small and the model had high forecast accuracy.
4.3. The Stress Prediction Model Based on Physical Model. According to the method described in other literature studies $[43,44]$, the material constants in equation (18) were finally obtained. The elastic stress of BT22 alloy was expressed as equation (33). The correlation coefficient between the experimental data and the predicted value is 0.98632 . So, equation (33) was suitable for fitting elastic stress of BT22 alloy.

$$
\sigma_{a}=1.533 *\left(e * \exp \left(\frac{2.079 * 10^{5}}{R * T}\right)\right)^{0.2045}
$$

The physical model was composed of elastic stress equation and dislocation density equation. Although the physical model had many parameters and the solution was complex, the internal changes of the alloy could be observed. In this paper, GA was used to identify the optimal model parameters to fit and predict the flow stress of BT22 alloy. The optimal solution of physical model parameters is given in Table 2.

Figure 7 (a) shows the scatter plot of the predicted value and experimental value of the training data. The predicted value and experimental value were in the same line, and the $R$ value and AARE value were $0.9842 \%$ and $5.9197 \%$, respectively. Figure 7(b) shows the scatter plot of the predicted and experimental value in validation data. The $R$ value and AARE value were 0.9714 and $7.2812 \%$, respectively, indicating that the physical model combined with GA had good prediction ability. Figures $7(\mathrm{c})$ and $7(\mathrm{~d})$ show the distribution of ARE values of the training set and verification set. The ARE values below $4 \%$ account for $50 \%$ and $47.7612 \%$, respectively. Nearly half of ARE values were higher than $4 \%$. The results show that the optimized physical equation could accurately describe the nonlinear flow behaviors of BT22 alloy, but the nonlinear prediction effect was not as good as the machine model.

4.4. Model Comparison. Table 3 compared the values of MSE value, $R$ value, and AARE value of the validation dataset, respectively. The results show that the four models had good 

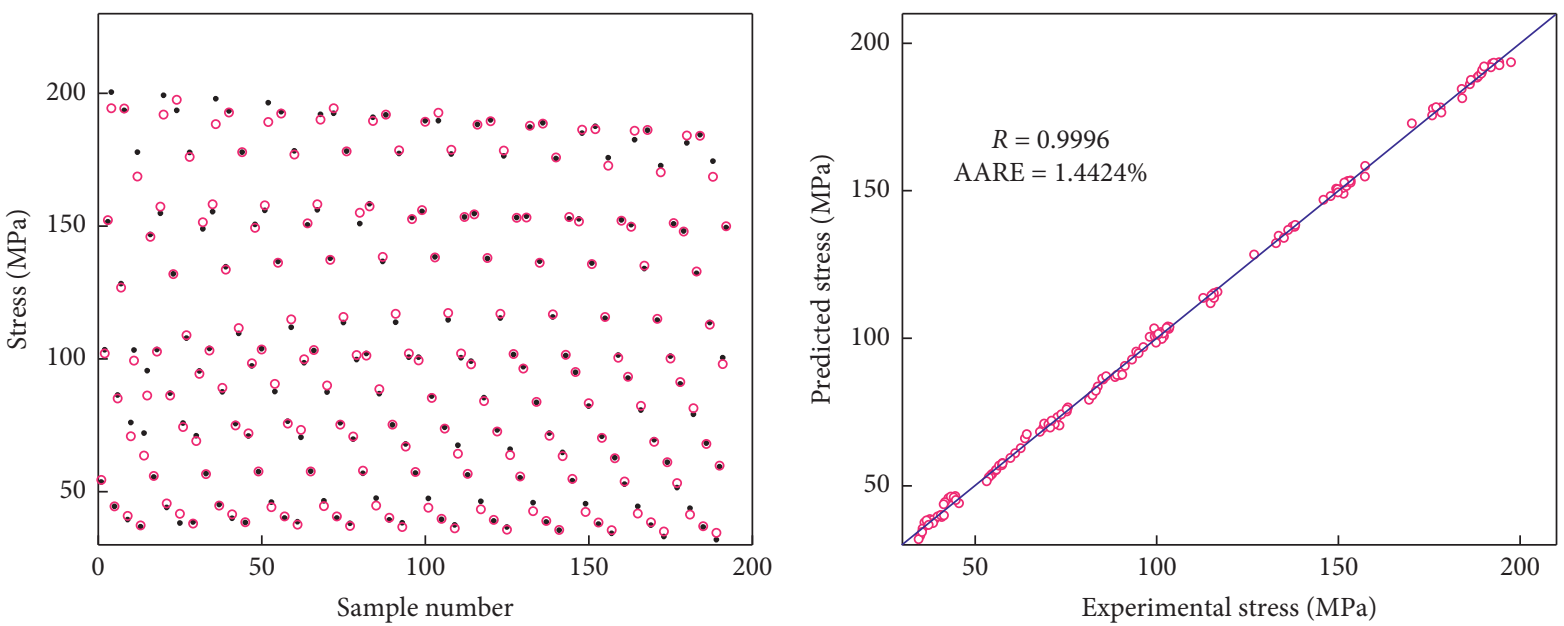

- Predicted stress

- Training data points

- Experimental stress

(a)

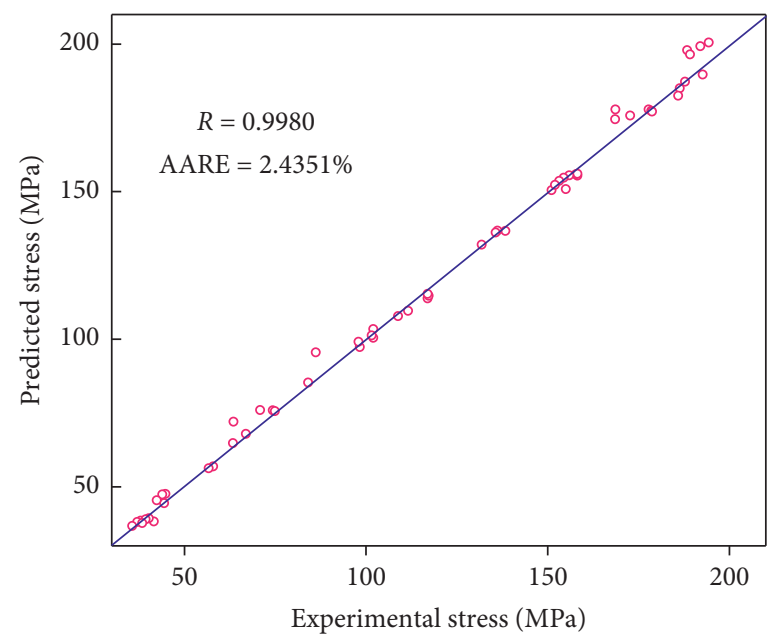

- Validation data points (b)

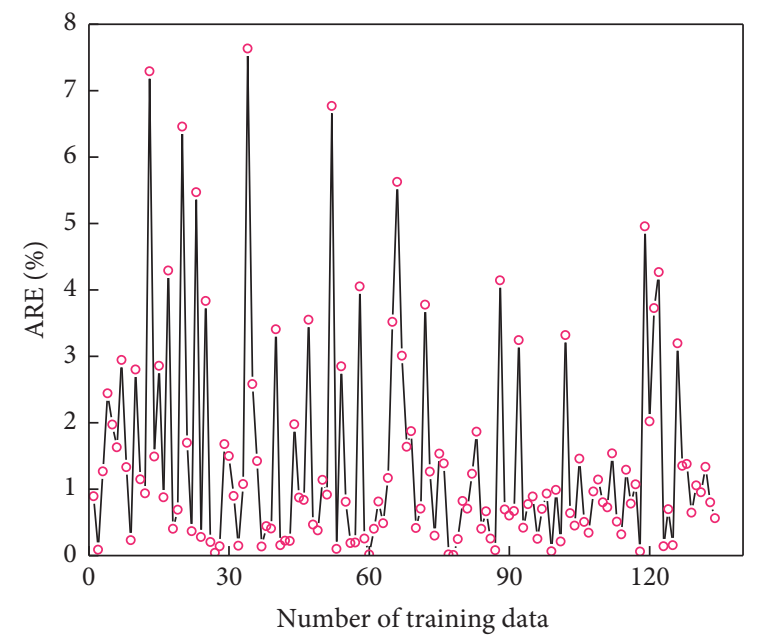

(d)

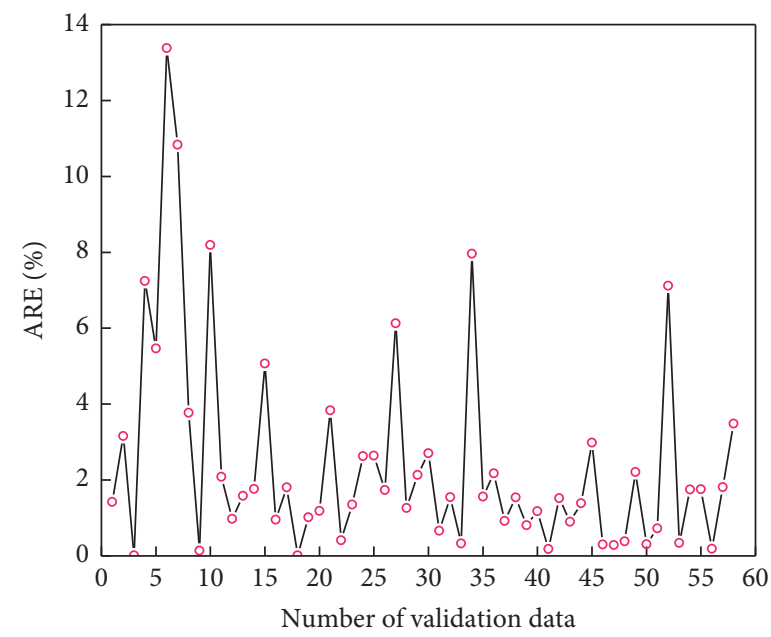

(e)

FIGURE 6: Correlations between the experimental stress and predicted stress by BPANN. (a) All data. (b) Training data. (c) Validation data. (d) ARE of training data. (e) ARE of validation data. 
Table 2: Parameters of the physical model.

\begin{tabular}{|c|c|c|c|c|c|c|}
\hline Model symbols & $A_{a}$ & $Q_{a}\left(\mathrm{~kJ} \mathrm{~mol}^{-1}\right)$ & $n_{a}$ & $A_{b}$ & $Q_{b}\left(\mathrm{~kJ} \mathrm{~mol}^{-1}\right)$ & $n_{b}$ \\
\hline Model coefficient & 128.211 & -72.578 & -1.445 & 3.53 & -25.6 & 1.127 \\
\hline
\end{tabular}

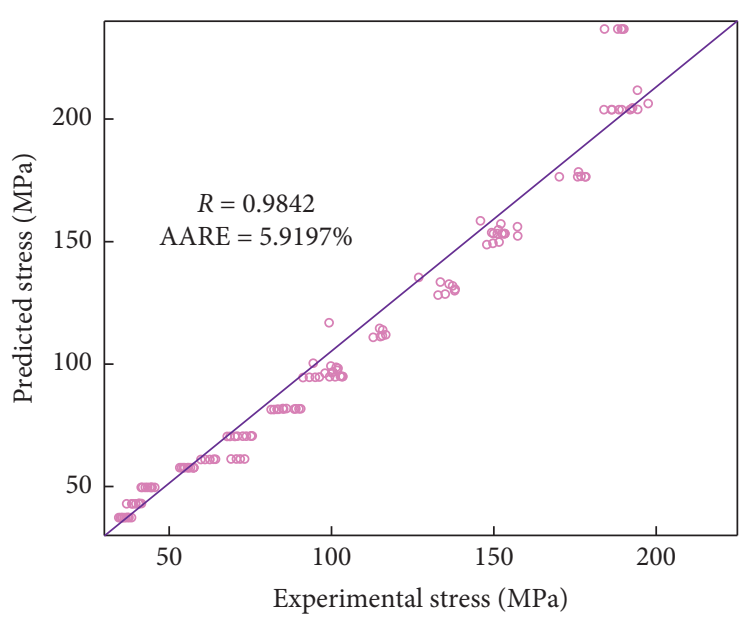

- Training data points

(a)

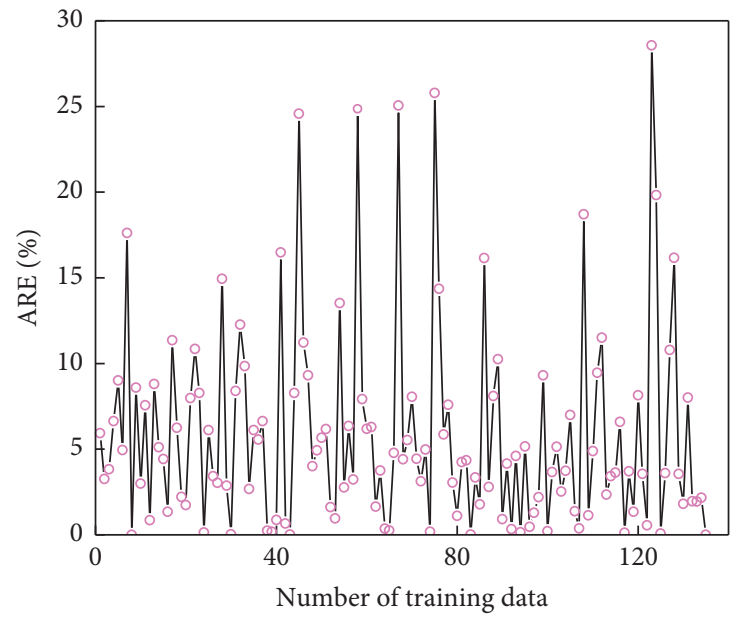

(c)

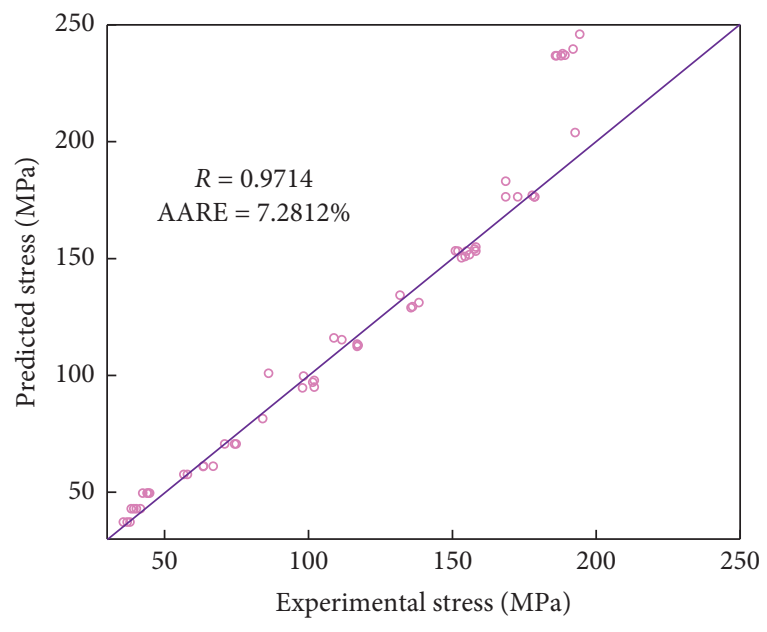

- Validation data points

(b)

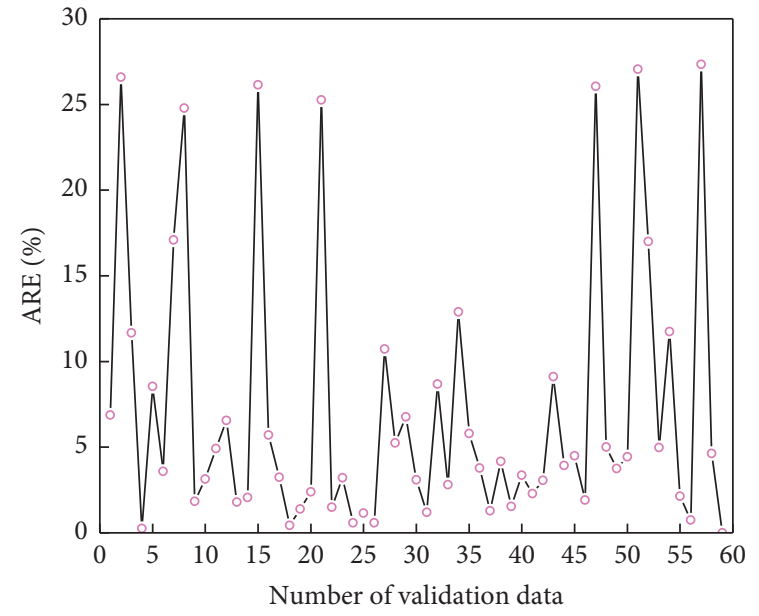

(d)

FIgURE 7: Correlations between the experimental stress and predicted stress by physical model. (a) Training data. (b) Validation data. (c) ARE of training data. (d) ARE of validation data.

prediction effect, but GA-SVR model had the best prediction accuracy. The MSE of GA-SVR model was $24.6804 \%$ lower than the SVR model, $15.3790 \%$ lower than the BPANN model, and $96.8185 \%$ lower than the physical model. Furthermore, the GA-SVR and BPANN model had higher prediction precision (MSE were both less than the SVR and physical model). GA-SVR and BPANN model were better choices for flow stress prediction, which were superior to the SVR and physical model.

Figures $8(a)-8(d)$ show the flow stress diagrams of the experimental stresses and predicted stresses of GA-SVR/ SVR/BPANN/physical model at strain rates of $0.01 \mathrm{~s}^{-1}$,
$0.1 \mathrm{~s}^{-1}, 1 \mathrm{~s}^{-1}$, and $10 \mathrm{~s}^{-1}$, respectively. As shown in Figure 8, some predicted values of the four models deviated from the experimental values, but they were all near the experimental curves. Compared with the SVR/BPANN/physical model, the predicted value of GA-SVR model was the closest to the experimental value and the deviation was the smallest.

As shown in Figure 9, the ARE values of BPANN, GASVR, and SVR model were volatile, and most of the ARE values were below $4 \%$ and accounted for $84.4828 \%$, $94.8276 \%$, and $82.7586 \%$, respectively. However, more than half of the ARE values in the physical model were higher than $4 \%$, accounting for $52.2388 \%$. Therefore, the GA-SVR 
TABLE 3: Comparison results of model prediction on the validation dataset for BT22 alloy.

\begin{tabular}{lccr}
\hline Model & AARE/\% & $R$ & MSE \\
\hline SVR & 2.2102 & 0.9979 & 13.4937 \\
GA-SVR & 1.5752 & 0.9984 & 10.1634 \\
BPANN & 2.4351 & 0.9980 & 12.0105 \\
Physical model & 7.2812 & 0.9714 & 319.4554 \\
\hline
\end{tabular}
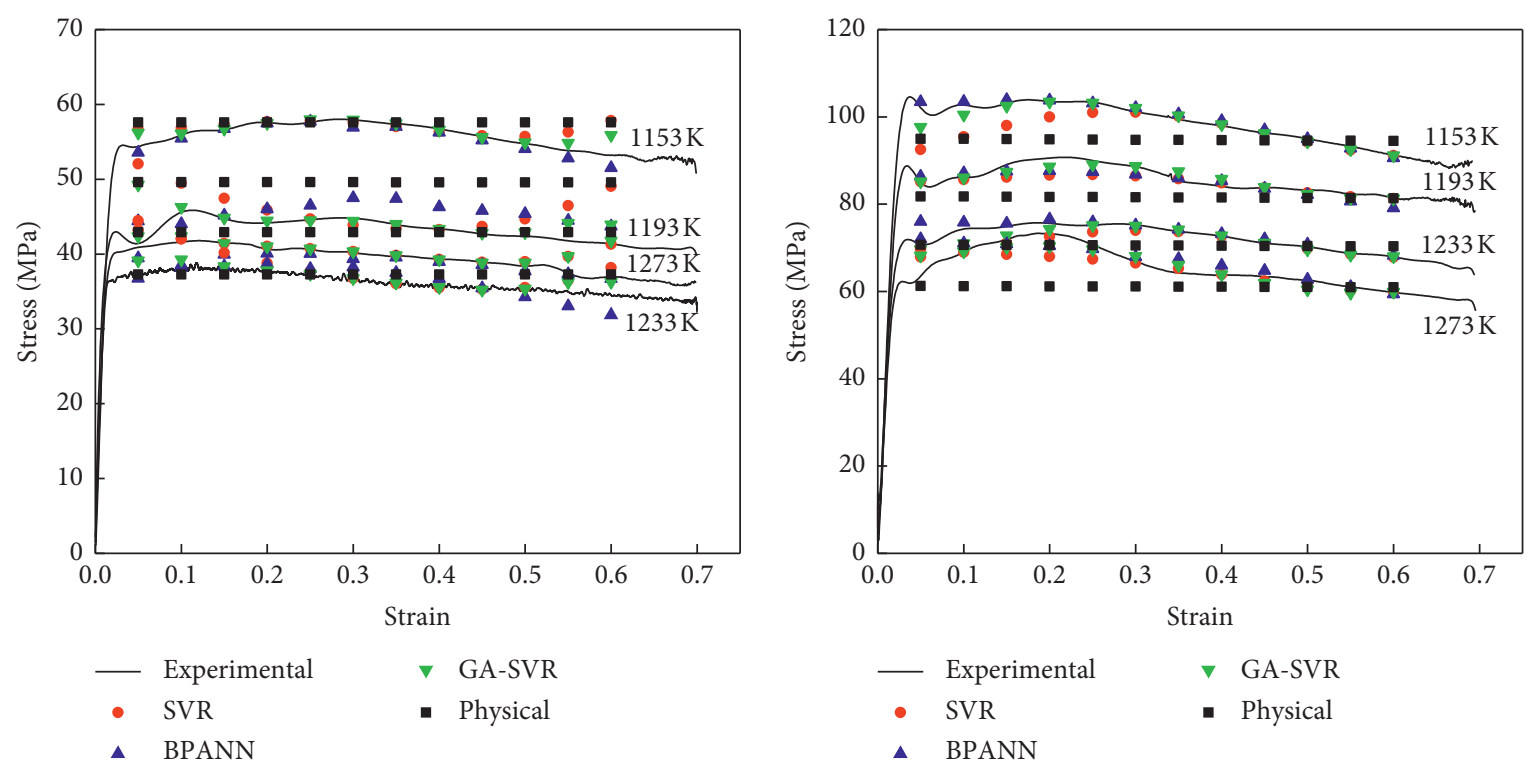

(a)

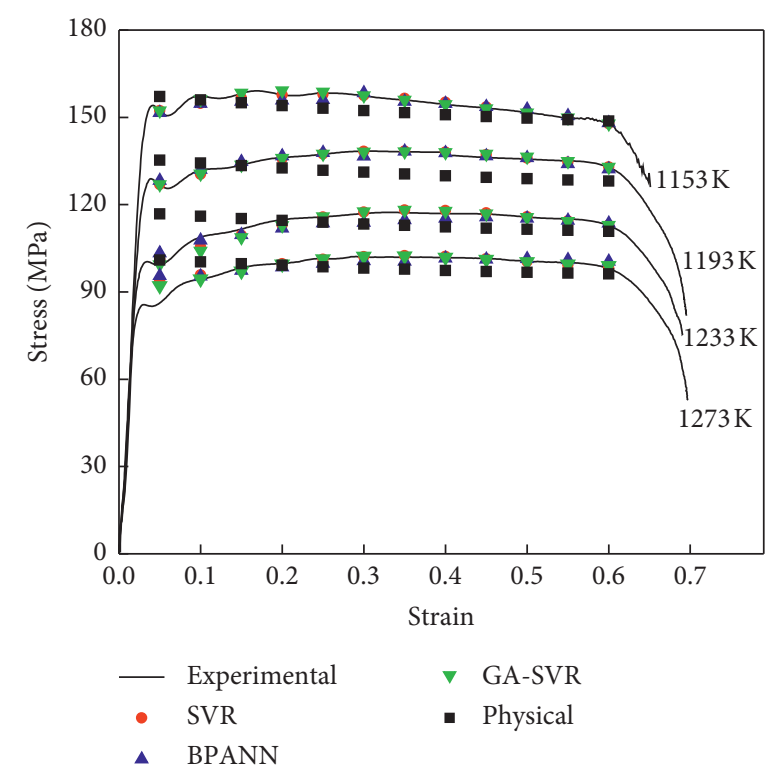

(c)

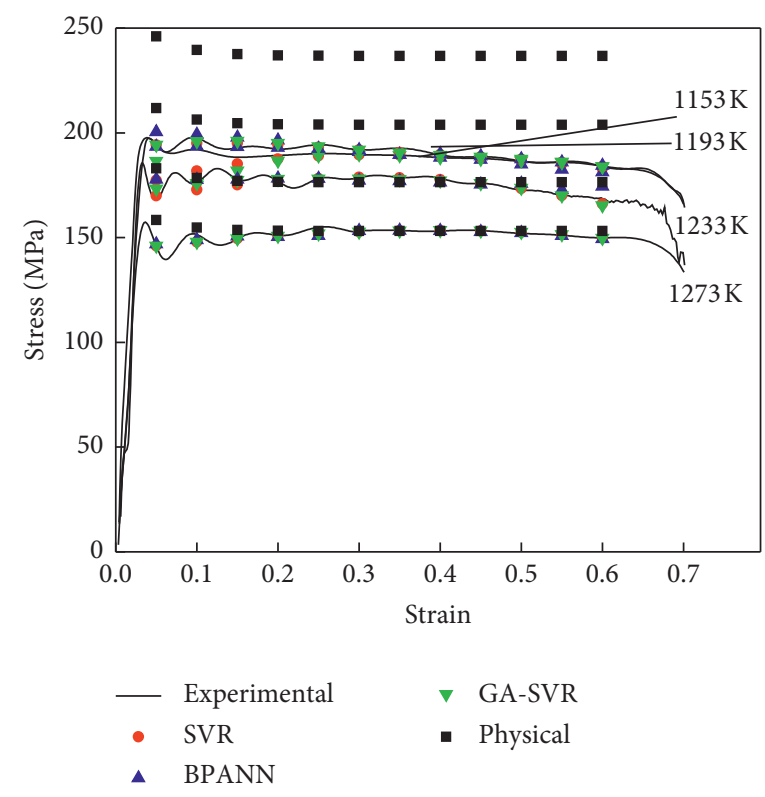

(d)

FIGURE 8: Comparison between the experimental and predicted flow stress by the physical model, SVR, BPANN, and GA-SVR model at different strain rates. (a) $0.01 \mathrm{~s}^{-1}$. (b) $0.1 \mathrm{~s}^{-1}$. (c) $1 \mathrm{~s}^{-1}$. (d) $10 \mathrm{~s}^{-1}$. 


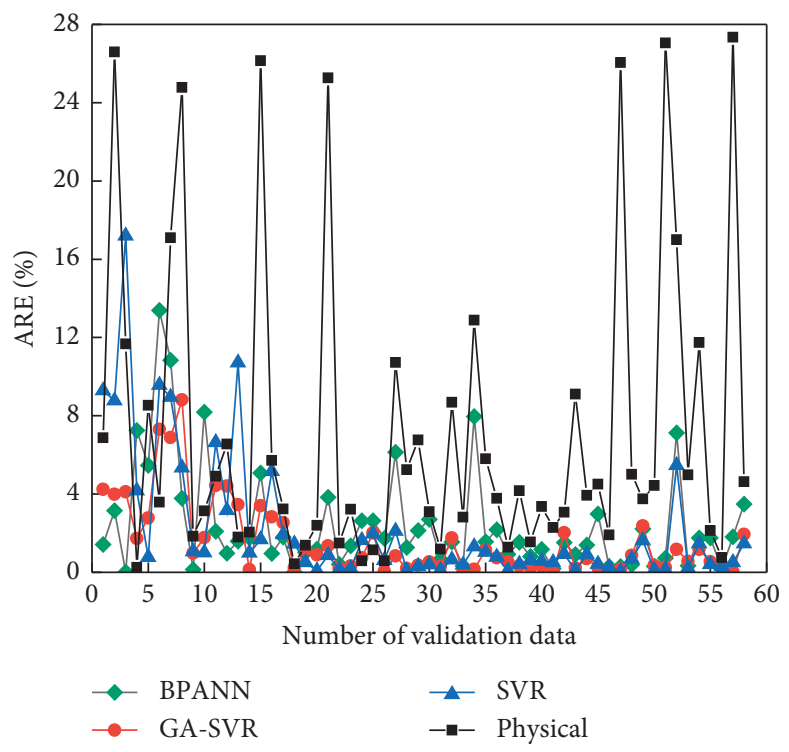

FIgURE 9: ARE value distribution of the four models.

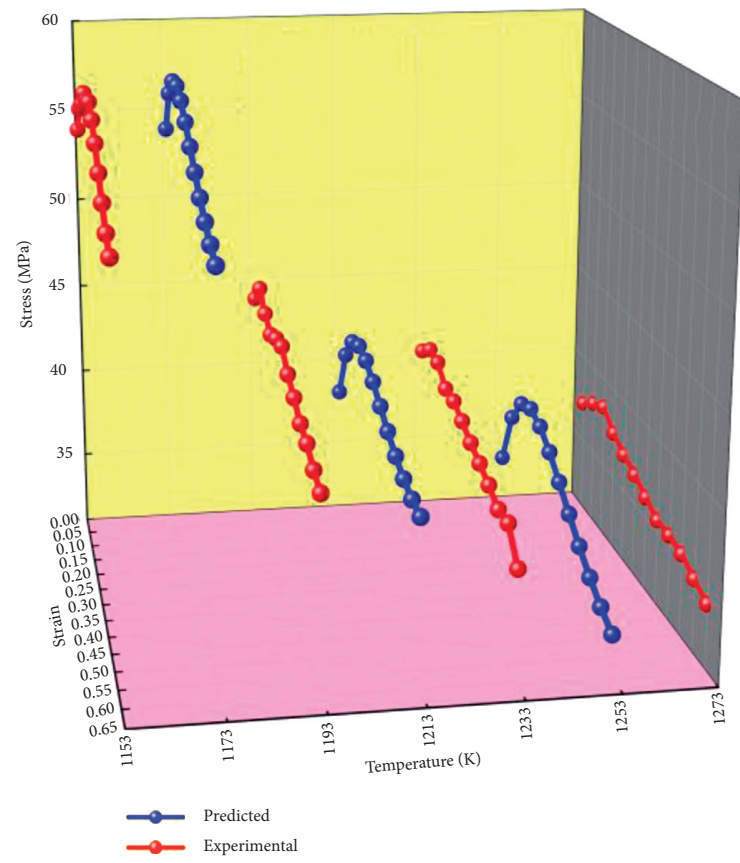

(a)

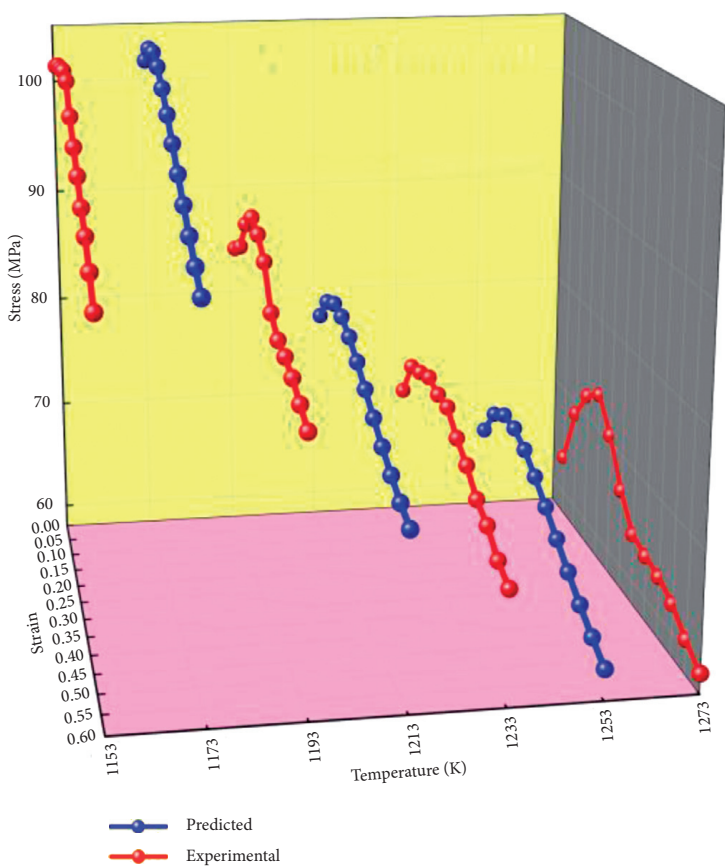

(b)

FIgure 10: Continued. 


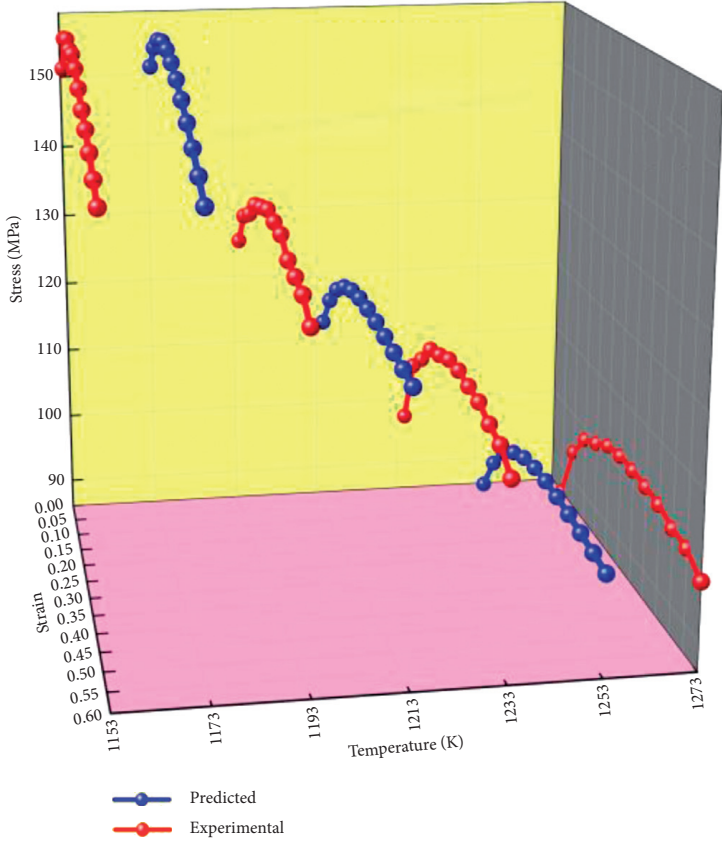

(c)

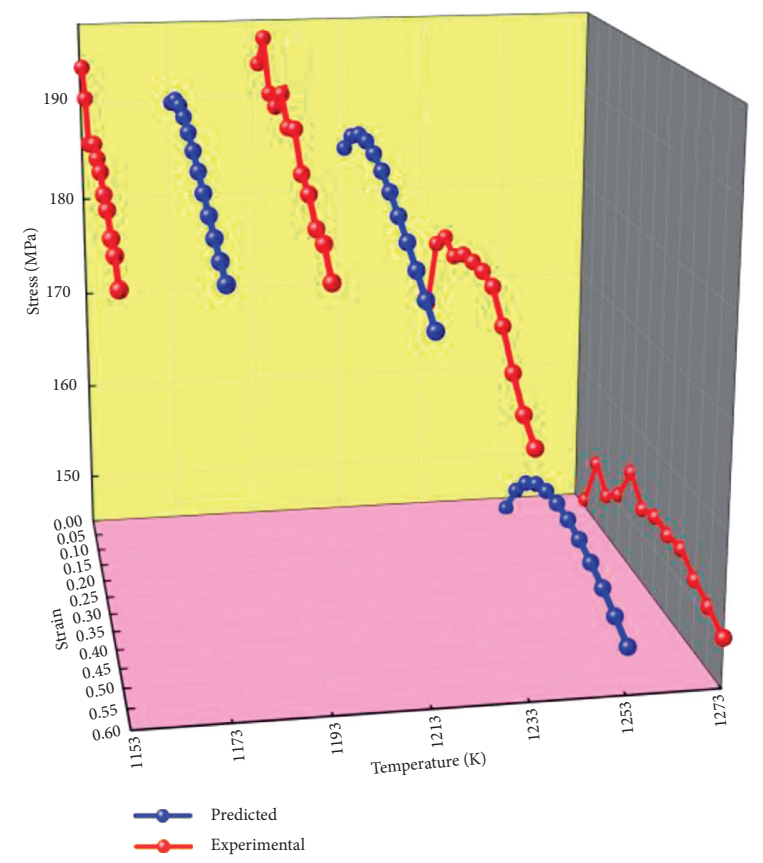

(d)

FIGURE 10: GA-SVR model predicts flow stress values under different conditions. (a) $0.01 \mathrm{~s}^{-1}$. (b) $0.1 \mathrm{~s}^{-1}$. (c) $1 \mathrm{~s}^{-1}$. (d) $10 \mathrm{~s}^{-1}$.

model had the best stability and fitting effect among four models, which had a good predictive ability in BT22 alloy. Quan et al. established Latin hypercube sampling with GA (LHS-GA), GA-SVR, ANN, and mathematical regression model to study the flow stress of Ti-10V-2Fe-3Al alloy at different conditions, respectively [7]. The results shown that the descending order of prediction accuracy was GASVR $>$ LHS-SVR > ANN > mathematical regression model. Chen et al. established the BPANN and regression model for Ti-6Al-3Nb-2Zr-1Mo alloy, with AARE of $1.42 \%$ and $6.53 \%$, respectively. Shi et al. studied the nonlinear flow stress of Ti-13Nb-13Zr alloy at different temperatures and strain rates. The AARE value of the GA-SVR model was always less than $0.18 \%$ whether it was $\beta$ or $\alpha+\beta$ phase region, and $R$ value was higher than 0.9999 [21]. By comparing the literature, this study had a similar conclusion that the prediction accuracy of GA-SVR model was better. The AARE value of the GA-SVR model is $1.5752 \%$, and the $R$ value is 0.9984 . GA-SVR model was used to predict the other deformation conditions (1173-1253 K), which greatly enriched the stress-strain data of BT22 alloy and reduced the number of tests.

\section{The Predicted Result of the GA-SVR Model}

By comparing the statistical indexes of the above four models, GA-SVR model had the highest stability and accuracy. Therefore, the GA-SVR model was used to predict the data without experimental simulation, which will better reflect the advantages of the model. Figures 10(a)-10(d) show the trend of flow stress at strain rates of $0.01 \mathrm{~s}^{-1}$, $0.1 \mathrm{~s}^{-1}, 1 \mathrm{~s}^{-1}$, and $10 \mathrm{~s}^{-1}$ for experimental values
(1153-1273 K) and predicted values (1173-1253 K). It can be seen from Figure 10 that the trend of predicted results (1173-1253 K) by the GA-SVR model was roughly the same as that of experimental values (1153-1273 K) at strain rates of $0.01,0.1,1$, and $10 \mathrm{~s}^{-1}$. Therefore, the GA-SVR model can be used to predict the nonlinear flow behaviors of BT22 alloy. At the same time, there was a close relationship between temperature, strain, strain rate, and flow stress in BT22 alloy.

\section{Conclusions}

Based on the stress-strain data at experimental conditions, the GA-SVR/SVR/BPANN/physical model was established to predict the nonlinear flow behaviors of BT22 alloy. Through the evaluation of the model, the following conclusions are drawn:

(1) In the aspect of model fitting ability, the AARE value of the GA-SVR model was lower than $1 \%$, which was $0.8388 \%$. However, the AARE values of SVR, BPANN, and physical models were higher than $1 \%$, and the $R$ values were lower than the GA-SVR model. The results show that the learning ability of SVR model after optimizing model parameters with GA was much higher than the other three models.

(2) In the aspect of model prediction ability, GA-SVR/ SVR/BPANN/physical models were evaluated by AARE, $R$, and ARE. The evaluation results show that AARE values of GA-SVR/SVR/BPANN/physical models were $1.5752 \%, 2.2102 \%, 2.4351 \%$, and $7.2812 \%$, and the $R$ values were $0.9984,0.9979,0.9980$, and 
0.9714 , respectively. The ARE values of the four models were mostly between $0 \%$ and $4 \%, 0 \%-10 \%, 0 \%-8 \%$, and $0-26 \%$ about accounting for $95 \%$. Therefore, the order of prediction accuracy of the four models was GA-SVR $>$ BPANN $>$ SVR $>$ physical model.

(3) The GA-SVR model was used to accurately predict the flow stress of BT22 alloy under different experimental conditions (not only under experimental conditions but also under nonexperimental conditions), which could expand the experimental stressstrain data and avoid a large number of artificial tests.

\section{Data Availability}

The data used to support the findings of this study are included in the article.

\section{Conflicts of Interest}

The authors declare that they have no conflicts of interest.

\section{Acknowledgments}

This work was financially supported by the Natural Science Foundation of Guangxi (no. 2019GXNSFBA245056).

\section{References}

[1] S. Y. Sun and W. J. Lv, "Microstructure and mechanical properties of TC18 titanium alloy," Rare Metal Materials \& Engineering, vol. 45, no. 5, pp. 1138-1441, 2016.

[2] S. Baragetti and F. Villa, "Corrosion fatigue of high-strength titanium alloys under different stress gradients," JOM, vol. 67, no. 5, pp. 1154-1161, 2015.

[3] M. Wang, J. Zhou, Y. Yin, H. Nan, P. Xue, and Z. Tu, "Hot deformation behavior of the Ti6Al4V alloy prepared by powder hot isostatic pressing," Journal of Alloys and Compounds, vol. 721, pp. 320-332, 2017.

[4] R. R. Boyer and R. D. Briggs, "The use of $\beta$ titanium alloys in the aerospace industry," Journal of Materials Engineering \& Performance, vol. 22, no. 10, pp. 2916-2920, 2013.

[5] G.-Z. Quan, S.-A. Pu, Z.-Y. Zhan, Z.-Y. Zou, Y.-Y. Liu, and Y.-F. Xia, "Modelling of the hot flow behaviors for Ti-13Nb$13 \mathrm{Zr}$ alloy by BP-ANN model and its application," International Journal of Precision Engineering and Manufacturing, vol. 16, no. 10, pp. 2129-2137, 2015.

[6] S. Long, Y.-F. Xia, P. Wang et al., "Constitutive modelling, dynamic globularization behavior and processing map for Ti6Cr-5Mo-5V-4Al alloy during hot deformation," Journal of Alloys and Compounds, vol. 796, pp. 65-76, 2019.

[7] G. Z. Quan, Z. H. Zhang, L. Zhang et al., "“Numerical descriptions of hot flow behaviors across $\beta$ transus for as-forged Ti-10V-2Fe-3Al alloy by LHS-SVR and GA-SVR and improvement in forming simulation accuracy," Applied Sciences, vol. 6, no. 8, Article ID 6080210, 2016.

[8] N. Kotkunde, A. D. Deole, A. K. Gupta, and S. K. Singh, "Comparative study of constitutive modeling for Ti-6Al-4V alloy at low strain rates and elevated temperatures," Materials \& Design, vol. 55, no. 3, pp. 999-1005, 2014.

[9] Y. C. Lin, Q.-F. Li, Y.-C. Xia, and L.-T. Li, "A phenomenological constitutive model for high temperature flow stress prediction of Al-Cu-Mg alloy," Materials Science and Engineering: A, vol. 534, pp. 654-662, 2012.

[10] J. Luo, M. Li, X. Li, and Y. Shi, "Constitutive model for high temperature deformation of titanium alloys using internal state variables," Mechanics of Materials, vol. 42, no. 2, pp. 157-165, 2010.

[11] G.-Z. Quan, W.-Q. Lv, Y.-P. Mao, Y.-W. Zhang, and J. Zhou, "Prediction of flow stress in a wide temperature range involving phase transformation for as-cast Ti-6Al-2Zr-1Mo-1V alloy by artificial neural network," Materials \& Design, vol. 50, no. 9, pp. 51-61, 2013.

[12] Y. Q. Jiang, Y. C. Lin, G. D. Pang et al., "Constitutive model and processing maps for a Ti-55511 alloy in $\beta$ region," Advanced Engineering Materials, vol. 22, no. 9, Article ID 1900930, 2019.

[13] H. S. Chen, Y. Feng, F. J. Ma et al., "Isothermal compression flow stress prediction of Ti-6Al-3Nb-2Zr-1Mo alloy based on BP-ANN," Rare Metal Materials \& Engineering, vol. 45, no. 6, pp. 1549-1553, 2016.

[14] G. Z. Quan, J. Pan, and X. Wang, "Prediction of the hot compressive deformation behavior for superalloy nimonic 80A by BP-ANN model," Applied Sciences, vol. 6, no. 3, Article ID 6030066, 2016.

[15] H.-Y. Li, J.-D. Hu, D.-D. Wei, X.-F. Wang, and Y.-H. Li, "Artificial neural network and constitutive equations to predict the hot deformation behavior of modified 2.25Cr-1Mo steel," Materials \& Design, vol. 42, no. 12, pp. 192-197, 2012.

[16] Y. C. Lin, D. G. He, J. Chen et al., "Microstructural evolution and support vector regression model for an aged Ni-based superalloy during two-stage hot forming with stepped strain rates," Materials \& Design, vol. 154, no. 9, pp. 51-62, 2018.

[17] G.-Z. Quan, Z.-H. Zhang, Y. Zhou, T. Wang, and Y.-F. Xia, "Numerical description of hot flow behaviors at Ti-6Al-2Zr$1 \mathrm{Mo}-1 \mathrm{~V}$ alloy by GA-SVR and relative applications," Materials Research, vol. 19, no. 6, pp. 1253-1269, 2016.

[18] J. Liu, W. Zeng, Y. Lai, and Z. Jia, “Constitutive model of Ti17 titanium alloy with Lamellar-type initial microstructure during hot deformation based on orthogonal analysis," $M a$ terials Science and Engineering: A, vol. 597, pp. 387-394, 2014.

[19] L. Su, H. Liu, L. Jing, Z. Yu, W. Wang, and L. Zhou, "Flow stress characteristics and microstructure evolution during hot compression of Nb-47Ti alloy," Journal of Alloys and Compounds, vol. 797, pp. 735-743, 2019.

[20] H. Wang, E. Li, and G. Y. Li, “The least square support vector regression coupled with parallel sampling scheme metamodeling technique and application in sheet forming optimization," Materials \& Design, vol. 30, no. 5, pp. 1468-1479, 2009.

[21] Z.-Y. Shi, G.-Z. Quan, C. An, H.-M. Qiu, W.-Y. Wang, and Z.-H. Zhang, "Artificial intelligence model of complicated flow behaviors for $\mathrm{Ti}-13 \mathrm{Nb}-13 \mathrm{Zr}$ alloy and relevant applications," Transactions of Nonferrous Metals Society of China, vol. 29, no. 10, pp. 2090-2098, 2019.

[22] X.-F. Li and Z.-M. Lu, "Optimizing the controllability of arbitrary networks with genetic algorithm," Physica A: Statistical Mechanics and its Applications, vol. 447, pp. 422-433, 2016.

[23] A. Jenab, I. Sari Sarraf, D. E. Green, T. Rahmaan, and M. J. Worswick, "The use of genetic algorithm and neural network to predict rate-dependent tensile flow behaviour of AA5182-O sheets," Materials \& Design, vol. 94, no. 3, pp. 262-273, 2016.

[24] T. M. Dejong, D. Da Silva, J. Vos, and A. J. Escobar-Gutiérrez, "Using functional-structural plant models to study, 
understand and integrate plant development and ecophysiology," Annals of Botany, vol. 108, no. 6, pp. 987-989, 2011.

[25] B. Efron, "Estimating the error rate of a prediction rule: improvement on cross-validation," Publications of the American Statal Association, vol. 78, no. 382, pp. 316-331, 1983.

[26] E. Colman, W. Waegeman, B. De Baets, and V. Fievez, "Prediction of subacute ruminal acidosis based on milk fatty acids: a comparison of linear discriminant and support vector machine approaches for model development," Computers and Electronics in Agriculture, vol. 111, pp. 179-185, 2015.

[27] A. J. Smola and B. Schölkopf, "A tutorial on support vector regression," Statistics and Computing, vol. 14, no. 3, pp. 199-222, 2004.

[28] R. Collobert and S. Bengio, "SVMTorch: support vector machines for large-scale regression problems," Journal of Machine Learning Research, vol. 1, pp. 143-160, 2001.

[29] R. M. Mohamed, A. El-Baz, and A. A. Farag, "Probability density estimation using advanced support vector machines and the expectation maximization algorithm," Proceedings of World Academy of Ence Engineering \& Technology, vol. 1, no. 137, pp. 183-186, 2007.

[30] V. N. Vapnik and A. Y. Chervonenkis, "On the uniform convergence of relative frequencies of events to their probabilities," Theory of Probability \& its Applications, vol. 16, no. 2, pp. 264-280, 1971.

[31] J. Wang, "A single-layer model for far-infrared radiation drying of onion slices," Drying Technology, vol. 20, no. 10, pp. 1941-1953, 2002.

[32] C. A. Micchelli, "Interpolation of scattered data: distance matrices and conditionally positive definite functions," Constructive Approximation, vol. 2, no. 1, pp. 11-22, 1986.

[33] D. E. Rummelhart, G. E. Hinton, and R. J. Williams, "Leaning internal representations by back-propagating errors," Nature, vol. 323, no. 6088, pp. 318-362, 1986.

[34] Y. C. Lin, W.-Y. Dong, M. Zhou, D.-X. Wen, and D.-D. Chen, "A unified constitutive model based on dislocation density for an $\mathrm{Al}-\mathrm{Zn}-\mathrm{Mg}-\mathrm{Cu}$ alloy at time-variant hot deformation conditions," Materials Science and Engineering: A, vol. 718, pp. 165-172, 2018.

[35] Y. C. Lin, D.-X. Wen, Y.-C. Huang, X.-M. Chen, and X.-W. Chen, "A unified physically based constitutive model for describing strain hardening effect and dynamic recovery behavior of a Ni-based superalloy," Journal of Materials Research, vol. 30, no. 24, pp. 3784-3794, 2015.

[36] B. Babu and L.-E. Lindgren, "Dislocation density based model for plastic deformation and globularization of Ti-6Al-4V," International Journal of Plasticity, vol. 50, no. 50, pp. 94-108, 2013.

[37] S. Mandal, B. T. Gockel, S. Balachandran, D. Banerjee, and A. D. Rollett, "Simulation of plastic deformation in Ti-5553 alloy using a self-consistent viscoplastic model," International Journal of Plasticity, vol. 94, pp. 57-73, 2017.

[38] W. Xiao, B. Wang, Y. Wu, and X. Yang, "Constitutive modeling of flow behavior and microstructure evolution of AA7075 in hot tensile deformation," Materials Science and Engineering: A, vol. 712, pp. 704-713, 2018.

[39] H. Liang, H. Guo, Y. Ning et al., "Dynamic recrystallization behavior of Ti-5Al-5Mo-5V-1Cr-1Fe alloy," Materials \& Design, vol. 63, no. 11, pp. 798-804, 2014.

[40] H. Mecking and U. F. Kocks, "Kinetics of flow and strainhardening," Acta Metallurgica, vol. 29, no. 11, pp. 1865-1875, 1981.
[41] E. Farabi, A. Zarei-Hanzaki, and H. R. Abedi, "Processing map development through elaborating phenomenological and physical constitutive based models," Advanced Engineering Materials, vol. 18, no. 4, pp. 572-581, 2016.

[42] C. Shi, W. Mao, and X.-G. Chen, "Evolution of activation energy during hot deformation of AA7150 aluminum alloy," Materials Science and Engineering: A, vol. 571, no. 6, pp. 83-91, 2013.

[43] J. S. Kim, J. H. Kim, Y. T. Lee, C. G. Park, and C. S. Lee, "Microstructural analysis on boundary sliding and its accommodation mode during superplastic deformation of Ti6Al-4V alloy," Materials Science and Engineering: A, vol. 263, no. 2, pp. 272-280, 1999.

[44] D. L. Holt, "Dislocation cell formation in metals," Journal of Applied Physics, vol. 41, no. 8, pp. 3197-3201, 1970.

[45] Y. C. Lin, S.-C. Luo, X.-Y. Jiang, Y. Tang, and M.-S. Chen, "Hot deformation behavior of a Sr-modified Al-Si-Mg alloy: constitutive model and processing maps," Transactions of Nonferrous Metals Society of China, vol. 28, no. 4, pp. 592-603, 2018.

[46] D.-G. He, Y. C. Lin, M.-S. Chen, J. Chen, D.-X. Wen, and X.-M. Chen, "Effect of pre-treatment on hot deformation behavior and processing map of an aged nickel-based superalloy," Journal of Alloys and Compounds, vol. 649, pp. 1075-1084, 2015. 